\title{
Martin boundary for some symmetric Lévy processes
}

\author{
Panki Kim* Renming Song ${ }^{\dagger}$ and Zoran Vondraček
}

\begin{abstract}
In this paper we study the Martin boundary of open sets with respect to a large class of purely discontinuous symmetric Lévy processes in $\mathbb{R}^{d}$. We show that, if $D \subset \mathbb{R}^{d}$ is an open set which is $\kappa$-fat at a boundary point $Q \in \partial D$, then there is exactly one Martin boundary point associated with $Q$ and this Martin boundary point is minimal.
\end{abstract}

AMS 2010 Mathematics Subject Classification: Primary 60J50, 31C40; Secondary 31C35, 60J45, 60J75.

Keywords and phrases: symmetric Lévy process, subordinate Brownian motion, Martin boundary, Martin kernel, boundary Harnack principle, Green function

\section{Introduction}

The Martin boundary of an open set $D$ is an abstract boundary introduced in 1941 by Martin [26] so that every nonnegative classical harmonic function in $D$ can be written as an integral of the Martin kernel with respect to a finite measure on the Martin boundary. This integral representation is called a Martin representation. The concepts of Martin boundary and Martin kernel were extended to general Markov processes by Kunita and Watanabe [25] in 1965. In order for the Martin representation to be useful, one needs to have a better understanding of the Martin boundary, for instance, its relation with the Euclidean boundary. In 1970, Hunt and Wheeden [14] proved that, in the classical case, the Martin boundary of a bounded Lipschitz domain coincides with its Euclidean boundary. Subsequently, a lot of progress has been made in studying the Martin boundary in the classical case.

With the help of the boundary Harnack principle for rotationally invariant $\alpha$-stable $(\alpha \in(0,2))$ processes established in [2], it was proved in [3, 11, 27] that the Martin boundary, with respect to the rotationally invariant $\alpha$-stable process, of a bounded Lipschitz domain $D$ coincides with its Euclidean boundary and that any nonnegative harmonic function with respect to the killed rotationally invariant $\alpha$-stable process in $D$ can be written uniquely as an integral of the Martin kernel with respect to a finite measure on $\partial D$. In [29] this result was extended to bounded $\kappa$ fat open sets. The Martin boundary, with respect to truncated stable processes, of any roughly

\footnotetext{
${ }^{*}$ This work was supported by the National Research Foundation of Korea(NRF) grant funded by the Korea government(MEST) (NRF-2013R1A2A2A01004822)

${ }^{\dagger}$ Research supported in part by a grant from the Simons Foundation (208236)
} 
connected $\kappa$-fat open set was shown in [16] to coincide with its Euclidean boundary. In [17, the results of [29] were extended to a large class of purely discontinuous subordinate Brownian motions. For Martin boundary at infinity with respect to subordinate Brownian motions, see [23].

In this paper, we study the Martin boundary of open set $D \subset \mathbb{R}^{d}$ with respect to a large class of symmetric, not necessarily rotationally invariant, (transient) Lévy processes killed upon exiting $D$. We show that if $D$ is an open set and $D$ is $\kappa$-fat at a single point $Q \in \partial D$, then the Martin boundary associated with $Q$ consists of exactly one point and the corresponding Martin kernel is a minimal harmonic function. Another point is that, unlike [3, 11, 16, 17, 27, 29], the set $D$ is not necessarily bounded. In the case when $D$ is unbounded, we do not study the Martin boundary associated with infinite boundary points.

Now we describe the class of processes we are going to work with.

Throughout this paper, $r \mapsto j(r)$ is a strictly positive and non-increasing function on $(0, \infty)$ satisfying

$$
j(r) \leq c j(r+1) \quad \text { for } r \geq 1
$$

and $X=\left(X_{t}, \mathbb{P}_{x}\right)$ is a purely discontinuous symmetric Lévy process with Lévy exponent $\Psi_{X}(\xi)$ so that

$$
\mathbb{E}_{x}\left[e^{i \xi \cdot\left(X_{t}-X_{0}\right)}\right]=e^{-t \Psi_{X}(\xi)}, \quad t>0, x \in \mathbb{R}^{d}, \xi \in \mathbb{R}^{d}
$$

We assume that the Lévy measure of $X$ has a density $J_{X}$ such that

$$
\gamma_{1}^{-1} j(|y|) \leq J_{X}(y) \leq \gamma_{1} j(|y|), \quad \text { for all } y \in \mathbb{R}^{d},
$$

for some $\gamma_{1}>1$. Since $\int_{0}^{\infty} j(r)\left(1 \wedge r^{2}\right) r^{d-1} d r<\infty$ by (1.2), the function $x \rightarrow j(|x|)$ is the Lévy density of an isotropic unimodal Lévy process whose characteristic exponent is

$$
\Psi(|\xi|)=\int_{\mathbb{R}^{d}}(1-\cos (\xi \cdot y)) j(|y|) d y
$$

The Lévy exponent $\Psi_{X}$ can be written as

$$
\Psi_{X}(\xi)=\int_{\mathbb{R}^{d}}(1-\cos (\xi \cdot y)) J_{X}(y) d y
$$

and, clearly by (1.2), it satisfies

$$
\gamma_{1}^{-1} \Psi(|\xi|) \leq \Psi_{X}(\xi) \leq \gamma_{1} \Psi(|\xi|), \quad \text { for all } \xi \in \mathbb{R}^{d}
$$

The function $\Psi$ may be not increasing. However, if we put $\Psi^{*}(r):=\sup _{s \leq r} \Psi(s)$, then, by [4, Proposition 2] (cf. also [13, Proposition 1]), we have

$$
\Psi(r) \leq \Psi^{*}(r) \leq \pi^{2} \Psi(r)
$$

Thus by (1.4),

$$
\left(\pi^{2} \gamma_{1}\right)^{-1} \Psi^{*}(|\xi|) \leq \Psi_{X}(\xi) \leq \gamma_{1} \Psi^{*}(|\xi|), \quad \text { for all } \xi \in \mathbb{R}^{d}
$$


We will always assume that $\Psi$ satisfies the following scaling condition at infinity:

$(\mathbf{H})$ : There exist constants $0<\delta_{1} \leq \delta_{2}<1$ and $a_{1}, a_{2}>0$ such that

$$
a_{1} \lambda^{2 \delta_{1}} \Psi(t) \leq \Psi(\lambda t) \leq a_{2} \lambda^{2 \delta_{2}} \Psi(t), \quad \lambda \geq 1, t \geq 1
$$

Then by [4, (15) and Corollary 22], for every $R>0$, there exists $c=c(R)>1$ such that

$$
c^{-1} \frac{\Psi\left(r^{-1}\right)}{r^{d}} \leq j(r) \leq c \frac{\Psi\left(r^{-1}\right)}{r^{d}} \text { for } r \in(0, R] .
$$

Note that the class of purely discontinuous symmetric Lévy processes considered in this paper contains some of the purely discontinuous isotropic unimodal Lévy processes dealt with in [4].

Let us now formulate precisely the main result of this paper.

Definition 1.1 Let $D \subset \mathbb{R}^{d}$ be an open set and $Q \in \mathbb{R}^{d}$. We say that $D$ is $\kappa$-fat at $Q$ for some $\kappa \in$ $\left(0, \frac{1}{2}\right)$, if there exists $R>0$ such that for all $r \in(0, R]$, there is a ball $B\left(A_{r}(Q), \kappa r\right) \subset D \cap B(Q, r)$. The pair $(R, \kappa)$ is called the characteristics of the $\kappa$-fat open set $D$ at $Q$. We say that an open set $D$ is $\kappa$-fat with characteristics $(R, \kappa)$ if $D$ is $\kappa$-fat at $Q \in \partial D$ with characteristics $(R, \kappa)$ for all $Q \in \partial D$.

For $D \subset \mathbb{R}^{d}$ we denote by $\partial_{M} D$ the Martin boundary of $D$. A point $w \in \partial_{M} D$ is said to be associated with $Q$ if there is a sequence $\left(y_{n}\right)_{n \geq 1} \subset D$ converging to $w$ in the Martin topology and to $Q$ in the Euclidean topology. The set of Martin boundary points associated with $Q$ is denoted by $\partial_{M}^{Q} D$.

Theorem 1.2 Suppose that the assumption $(\mathbf{H})$ is satisfied. Let $X$ be a symmetric Lévy process with a Lévy density satisfying (1.2) and let $D$ be an open subset of $\mathbb{R}^{d}$ which is $\kappa$-fat at $Q \in \partial D$. If $D$ is bounded, then $\partial_{M}^{Q} D$ consists of exactly one point and this point is a minimal Martin boundary point. If $D$ is unbounded and the process $X$ is transient, the same conclusion is true.

In the case when $D$ is unbounded, a natural assumption would be that $D$ is Greenian, that is, the killed process $X^{D}$ is transient. Unfortunately, under the assumption $(\mathbf{H})$, which governs the behavior of the process in small space, it seems difficult to obtain estimates of the Green function $G_{D}(x, y)$ when either $x$ or $y$ becomes large. This is why in the case of unbounded $D$ we impose the transience assumption on $X$ which gives the asymptotic behavior of the free Green function $G(x, y)$, cf. Lemma 2.20.

We begin the paper by showing that only small modifications are needed to extend some results from the isotropic case studied in [20] to the symmetric Lévy processes $X$ considered in this paper. These results include exit time estimates, Poisson kernel estimates and Harnack inequality. A little more work is needed to establish the upper and the lower bound on the Green function $G_{D}$. Those are used to obtain sharp bounds on the Poisson kernel and the boundary Harnack principle in the same way as in [20]. In Section 3 we follow the well-established route, see [2, 17, 19, 23], to identify 
the Martin boundary point associated to $Q$. After preliminary estimates about harmonic functions, we first show that the oscillation reduction lemma, see [2, Lemma 16], is valid in our setting (with essentially the same proof). The lemma almost immediately implies that $\partial_{M}^{Q} D$ consists of exactly one point. We then show that this point is a minimal Martin boundary point. We end the paper by giving the Martin representation for bounded $\kappa$-fat open sets.

We finish this introduction by setting up some notation and conventions. We use ":=" to denote a definition, which is read as "is defined to be"; we denote $a \wedge b:=\min \{a, b\}, a \vee b:=\max \{a, b\}$; we denote by $B(x, r)$ the open ball centered at $x \in \mathbb{R}^{d}$ with radius $r>0$; for any two positive functions $f$ and $g, f \asymp g$ means that there is a positive constant $c \geq 1$ so that $c^{-1} g \leq f \leq c g$ on their common domain of definition; for any Borel subset $E \subset \mathbb{R}^{d}$ and $x \in E$, $\operatorname{diam}(E)$ stands for the diameter of $E$ and $\delta_{E}(x)$ stands for the Euclidean distance between $x$ and $E^{c} ; \mathbb{N}$ is the set of natural numbers.

In this paper, we use the following convention: The values of the constants $R, \delta_{1}, \delta_{2}, C_{1}, C_{2}, C_{3}, C_{4}$ remain the same throughout this paper, while $c, c_{0}, c_{1}, c_{2}, \ldots$ represent constants whose values are unimportant and may change. All constants are positive finite numbers. The labeling of the constants $c_{0}, c_{1}, c_{2}, \ldots$ starts anew in the statement and proof of each result. The dependence of constant $c$ on dimension $d$ is not mentioned explicitly.

\section{Green function estimates}

Let $S=\left(S_{t}: t \geq 0\right)$ be a subordinator with no drift. The Laplace exponent $\phi$ of $S$ is a Bernstein function and admits the following representation

$$
\phi(\lambda)=\int_{0}^{\infty}\left(1-e^{-\lambda t}\right) \mu(d t), \quad \lambda>0,
$$

where $\mu$ satisfies $\int_{0}^{\infty}(1 \wedge t) \mu(d t)<\infty$. $\mu$ is called the Lévy measure of $S$ or $\phi$. The function $\phi$ is called a complete Bernstein function if $\mu$ has a completely monotone density.

The following elementary result observed in [19, 22] will be used several times later in this paper.

Lemma 2.1 If $\phi$ is a Bernstein function, then for all $\lambda, t>0,1 \wedge \lambda \leq \phi(\lambda t) / \phi(t) \leq 1 \vee \lambda$.

Recall that, throughout this paper, we assume that $X=\left(X_{t}, \mathbb{P}_{x}\right)$ is a purely discontinuous symmetric Lévy process in $\mathbb{R}^{d}$ with Lévy exponent $\Psi_{X}(\xi)$ and a Lévy density $J_{X}$ satisfying (1.2).

It follows from [4, (28)], (1.4), (1.6) and (1.7) that there exist a constant $\gamma_{2}>1$ and a complete Bernstein function $\phi$ such that

$$
\gamma_{2}^{-1} \phi\left(|\xi|^{2}\right) \leq \Psi(|\xi|) \leq \gamma_{2} \phi\left(|\xi|^{2}\right), \quad \text { for all } \xi \in \mathbb{R}^{d}
$$

and $j$ enjoys the following property: for every $R>0$,

$$
j(r) \asymp \frac{\phi\left(r^{-2}\right)}{r^{d}} \text { for } r \in(0, R] .
$$


Furthermore, there exist $b_{1}, b_{2}>0$ such that

$$
b_{1} \lambda^{\delta_{1}} \phi(t) \leq \phi(\lambda t) \leq b_{2} \lambda^{\delta_{2}} \phi(t), \quad \lambda \geq 1, t \geq 1 .
$$

Throughout this paper, we assume that $\phi$ is the above complete Bernstein function.

From Lemma 2.1 and (2.2) , we also get that for every $R>0$,

$$
j(r) \leq c j(2 r), \quad r \in(0, R] .
$$

The infinitesimal generator $\mathbf{L}$ of $X$ is given by

$$
\mathbf{L} f(x)=\int_{\mathbb{R}^{d}}\left(f(x+y)-f(x)-y \cdot \nabla f(x) \mathbf{1}_{\{|y| \leq 1\}}\right) J_{X}(y) d y
$$

for $f \in C_{b}^{2}\left(\mathbb{R}^{d}\right)$. Furthermore, for every $f \in C_{b}^{2}\left(\mathbb{R}^{d}\right), f\left(X_{t}\right)-f\left(X_{0}\right)-\int_{0}^{t} \mathbf{L} f\left(X_{s}\right) d s$ is a $\mathbb{P}_{x^{-}}$martingale for every $x \in \mathbb{R}^{d}$.

The following two results are valid without assuming $(\mathbf{H})$. The next lemma is a special case of [13, Corollary 1].

Lemma 2.2 There exists a constant $c>0$ depending only on $d$ such that

$$
c^{-1} \Psi\left(r^{-1}\right) \leq \frac{1}{r^{2}} \int_{0}^{r} s^{d+1} j(s) d s+\int_{r}^{\infty} s^{d-1} j(s) d s \leq c \Psi\left(r^{-1}\right), \quad \forall r>0 .
$$

Lemma 2.3 There exists a constant $c=c\left(\Psi, \gamma_{1}, \gamma_{2}\right)>0$ such that for every $f \in C_{b}^{2}\left(\mathbb{R}^{d}\right)$ with $0 \leq f \leq 1$

$$
\mathbf{L} f_{r}(x) \leq c \phi\left(r^{-2}\right)\left(2+\sup _{y} \sum_{j, k}\left|\left(\partial^{2} / \partial y_{j} \partial y_{k}\right) f(y)\right|\right), \quad \text { for every } x \in \mathbb{R}^{d}, r>0,
$$

where $f_{r}(y):=f(y / r)$.

Proof. Using (1.2) and Lemma 2.2 or [4, Corollary 3], this result can be obtained by following the proof of [21, Lemma 4.2]. We omit the details.

For any open set $D$, we use $\tau_{D}$ to denote the first exit time of $D$, i.e., $\tau_{D}=\inf \left\{t>0: X_{t} \notin D\right\}$.

Using Lemmas 2.1 and 2.3 and (2.1), the proof of the next result is the same as those of [18, Lemmas 13.4.1 and 13.4.2]. Thus we omit the proof.

Lemma 2.4 There exists a constant $c=c\left(\Psi, \gamma_{1}, \gamma_{2}\right)>0$ such that for every $r>0$ and every $x \in \mathbb{R}^{d}$,

$$
\inf _{z \in B(x, r / 2)} \mathbb{E}_{z}\left[\tau_{B(x, r)}\right] \geq \frac{c}{\phi\left(r^{-2}\right)}
$$

The idea of the following key result comes from [31]. 
Lemma 2.5 There exists a constant $c=c\left(\Psi, \gamma_{1}, \gamma_{2}\right)>0$ such that for any $r>0$ and $x_{0} \in \mathbb{R}^{d}$,

$$
\mathbb{E}_{x}\left[\tau_{B\left(x_{0}, r\right)}\right] \leq c\left(\phi\left(r^{-2}\right) \phi\left(\left(r-\left|x-x_{0}\right|\right)^{-2}\right)\right)^{-1 / 2}, \quad x \in B\left(x_{0}, r\right) .
$$

Proof. Without loss of generality, we may assume that $x_{0}=0$. We fix $x \neq 0$ and put $Z_{t}=\frac{X_{t} \cdot x}{|x|}$. Then, $Z_{t}$ is a one dimensional symmetric Lévy process in $\mathbb{R}$ with Lévy exponent

$$
\Psi_{Z}(\theta)=\int_{\mathbb{R}^{d}}\left(1-\cos \left(\frac{\theta x}{|x|} \cdot y\right)\right) J_{X}(y) d y, \quad \theta \in \mathbb{R} .
$$

By (1.2),

$$
\Psi_{Z}(\theta) \asymp \int_{\mathbb{R}^{d}}\left(1-\cos \left(\frac{\theta x}{|x|} \cdot y\right)\right) j(|y|) d y=\Psi(\theta) .
$$

It is easy to see that, if $X_{t} \in B(0, r)$, then $\left|Z_{t}\right|<r$, hence $\mathbb{E}_{x}\left[\tau_{B(0, r)}\right] \leq \mathbb{E}_{|x|}[\tilde{\tau}]$, where $\tilde{\tau}=$ $\inf \left\{t>0:\left|Z_{t}\right| \geq r\right\}$. By [5, (2.17)], the proof of [5, Proposition 2.4] and Lemma 2.2.

$$
\mathbb{E}_{x}\left[\tau_{B(0, r)}\right] \leq \mathbb{E}_{|x|}[\tilde{\tau}] \leq c\left(\Psi\left(r^{-1}\right) \Psi\left((r-|x|)^{-1}\right)\right)^{-1 / 2} .
$$

Now the assertion of the lemma follows immediately by (2.1).

Given an open set $D \subset \mathbb{R}^{d}$, we define $X_{t}^{D}(\omega)=X_{t}(\omega)$ if $t<\tau_{D}(\omega)$ and $X_{t}^{D}(\omega)=\partial$ if $t \geq \tau_{D}(\omega)$, where $\partial$ is a cemetery state. We now recall the definitions of harmonic functions with respect to $X$ and with respect to $X^{D}$.

Definition 2.6 Let $D$ be an open subset of $\mathbb{R}^{d}$. A nonnegative function $u$ on $\mathbb{R}^{d}$ is said to be

(1) harmonic in $D$ with respect to $X$ if

$$
u(x)=\mathbb{E}_{x}\left[u\left(X_{\tau_{U}}\right)\right], \quad x \in U,
$$

for every open set $U$ whose closure is a compact subset of $D$;

(2) regular harmonic in $D$ with respect to $X$ if for each $x \in D$,

$$
u(x)=\mathbb{E}_{x}\left[u\left(X_{\tau_{D}}\right) ; \tau_{D}<\infty\right] .
$$

Definition 2.7 Let $D$ be an open subset of $\mathbb{R}^{d}$. A nonnegative function $u$ on $D$ is said to be harmonic with respect to $X^{D}$ if

$$
u(x)=\mathbb{E}_{x}\left[u\left(X_{\tau_{U}}\right)\right], \quad x \in U,
$$

for every open set $U$ whose closure is a compact subset of $D$.

Obviously, if $u$ is harmonic with respect to $X^{D}$, then the function which is equal to $u$ in $D$ and zero outside $D$ is harmonic with respect to $X$ in $D$.

Since our $X$ satisfies [7, (1.6), (UJS)], by [7, Theorem 1.4] and using the standard chain argument one has the following form of Harnack inequality. 
Theorem 2.8 For every $a \in(0,1)$, there exists $c=c\left(a, \Psi, \gamma_{1}, \gamma_{2}\right)>0$ such that for every $r \in(0,1)$, $x_{0} \in \mathbb{R}^{d}$, and every function $u$ which is nonnegative on $\mathbb{R}^{d}$ and harmonic with respect to $X$ in $B\left(x_{0}, r\right)$, we have

$$
u(x) \leq c u(y), \quad \text { for all } x, y \in B\left(x_{0}, a r\right) .
$$

Let $D \subset \mathbb{R}^{d}$ be an open set. Since $J_{X}$ satisfies the assumption [7, (1.6)], by [7, Theorem 3.1], bounded functions that are harmonic in $D$ with respect to $X$ are Hölder continuous. Suppose that $u$ is a nonnegative function which is harmonic with respect to $X$ in $D$. For any ball $B:=B\left(x_{0}, r\right)$ with $B \subset \bar{B} \subset D$, the functions $u_{n}, n \geq 1$, defined by

$$
u_{n}(x):=\mathbb{E}_{x}\left[(u \wedge n)\left(X_{\tau_{B}}\right)\right], \quad x \in \mathbb{R}^{d},
$$

are bounded functions which are harmonic with respect to $X$ in $B$. Applying Theorem 2.8 to $v_{n}(x):=u(x)-u_{n}(x)=\mathbb{E}_{x}\left[(u-(u \wedge n))\left(X_{\tau_{B}}\right)\right]$, it is easy to see that $u_{n}$ converges to $u$ uniformly in $B\left(x_{0}, r / 2\right)$. Thus $u$ is continuous in $D$. This implies that all nonnegative functions that are harmonic in $D$ with respect to $X$ are continuous.

A subset $D$ of $\mathbb{R}^{d}$ is said to be Greenian (for $X$ ) if $X^{D}$ is transient. By [7, Theorem 3.1] $X^{D}$ has Hölder continuous transition densities $p_{D}(t, x, y)$. For any Greenian open set $D$ in $\mathbb{R}^{d}$, let $G_{D}(x, y)=\int_{0}^{\infty} p_{D}(t, x, y) d t$ be the Green function of $X^{D}$. Then $G_{D}(x, y)$ is finite off the diagonal $D \times D$. Furthermore, $x \mapsto G_{D}(x, y)$ is harmonic in $D \backslash\{y\}$ with respect to $X$ and therefore continuous. Using the Lévy system for $X$, we know that for every Greenian open subset $D$ and every $f \geq 0$ and $x \in D$,

$$
\mathbb{E}_{x}\left[f\left(X_{\tau_{D}}\right) ; X_{\tau_{D}-} \neq X_{\tau_{D}}\right]=\int_{\bar{D}^{c}} \int_{D} G_{D}(x, z) J_{X}(z-y) d z f(y) d y .
$$

We define the Poisson kernel

$$
K_{D}(x, y):=\int_{D} G_{D}(x, z) J_{X}(z-y) d z, \quad(x, y) \in D \times \bar{D}^{c} .
$$

Thus (2.7) can be simply written as

$$
\mathbb{E}_{x}\left[f\left(X_{\tau_{D}}\right) ; X_{\tau_{D}-} \neq X_{\tau_{D}}\right]=\int_{\bar{D}^{c}} K_{D}(x, y) f(y) d y .
$$

The following result will be used later in this paper.

Lemma 2.9 There exist $c_{1}=c_{1}\left(\Psi, \gamma_{1}, \gamma_{2}\right)>0$ and $c_{2}=c_{2}\left(\Psi, \gamma_{1}, \gamma_{2}\right)>0$ such that for every $r \in(0,1]$ and $x_{0} \in \mathbb{R}^{d}$,

$$
\begin{aligned}
K_{B\left(x_{0}, r\right)}(x, y) & \leq c_{1} j\left(\left|y-x_{0}\right|-r\right)\left(\phi\left(r^{-2}\right) \phi\left(\left(r-\left|x-x_{0}\right|\right)^{-2}\right)\right)^{-1 / 2} \\
& \leq c_{1} j\left(\left|y-x_{0}\right|-r\right) \phi\left(r^{-2}\right)^{-1}
\end{aligned}
$$

for all $(x, y) \in B\left(x_{0}, r\right) \times{\overline{B\left(x_{0}, r\right)}}^{c}$ and

$$
K_{B\left(x_{0}, r\right)}\left(x_{0}, y\right) \geq c_{2} j\left(\left|y-x_{0}\right|\right) \phi\left(r^{-2}\right)^{-1}, \quad \text { for all } y \in{\overline{B\left(x_{0}, r\right)}}^{c} .
$$


Proof. This proof is exactly the same as that of [18, Proposition 13.4.10]. We provide the proof to show that only the monotonicity of $j$, (1.1), (2.4) and Lemmas 2.4 and 2.5 are used.

Without loss of generality, we assume $x_{0}=0$. For $z \in B(0, r)$ and $r<|y|<2$,

$$
|y|-r \leq|y|-|z| \leq|y-z| \leq|z|+|y|+r+|y| \leq 2|y|
$$

and for $z \in B(0, r)$ and $y \in B(0,2)^{c}$,

$$
|y|-r \leq|y|-|z| \leq|y-z| \leq|z|+|y|+r+|y| \leq|y|+1 \text {. }
$$

Thus by the monotonicity of $j,(1.1)$ and (2.4), there exists a constant $c>0$ such that

$$
c j(|y|) \leq j(|z-y|) \leq j(|y|-r), \quad(z, y) \in B(0, r) \times \overline{B(0, r)}^{c} .
$$

Applying the above inequalities, Lemmas 2.4 and 2.5 to (2.8), we immediately get the assertion of the lemma.

As in [20], to deal with $\kappa$-fat open set, we need the following form of Harnack inequality.

Theorem 2.10 Let $L>0$. There exists a positive constant $c=c\left(L, \Psi, \gamma_{1}, \gamma_{2}\right)>1$ such that the following is true: If $x_{1}, x_{2} \in \mathbb{R}^{d}$ and $r \in(0,1)$ are such that $\left|x_{1}-x_{2}\right|<L r$, then for every nonnegative function $u$ which is harmonic with respect to $X$ in $B\left(x_{1}, r\right) \cup B\left(x_{2}, r\right)$, we have

$$
c^{-1} u\left(x_{2}\right) \leq u\left(x_{1}\right) \leq c u\left(x_{2}\right) .
$$

Proof. Let $r \in(0,1], x_{1}, x_{2} \in \mathbb{R}^{d}$ be such that $\left|x_{1}-x_{2}\right|<L r$ and let $u$ be a nonnegative function which is harmonic in $B\left(x_{1}, r\right) \cup B\left(x_{2}, r\right)$ with respect to $X$. If $\left|x_{1}-x_{2}\right|<\frac{1}{4} r$, then since $r<1$, the theorem is true by Theorem 2.8. Thus we only need to consider the case when $\frac{1}{4} r \leq\left|x_{1}-x_{2}\right| \leq L r$ with $L>\frac{1}{4}$.

Let $w \in B\left(x_{1}, \frac{r}{8}\right)$. Because $\left|x_{2}-w\right| \leq\left|x_{1}-x_{2}\right|+\left|w-x_{1}\right|<\left(L+\frac{1}{8}\right) r \leq 2 L r$, first using the monotonicity of $j$ and (2.10), then using (2.2) and Lemma 2.1, we get

$$
K_{B\left(x_{2}, \frac{r}{8}\right)}\left(x_{2}, w\right) \geq c_{1} j(2 L r) \phi\left(r^{-2}\right)^{-1} \geq c_{2} r^{-d} \frac{\phi\left((2 L r)^{-2}\right)}{\phi\left(r^{-2}\right)} \geq c_{3} r^{-d} .
$$

For any $y \in B\left(x_{1}, \frac{r}{8}\right), u$ is regular harmonic in $B\left(y, \frac{7 r}{8}\right) \cup B\left(x_{1}, \frac{7 r}{8}\right)$. Since $\left|y-x_{1}\right|<\frac{r}{8}$, by the already proven part of this theorem,

$$
u(y) \geq c_{2} u\left(x_{1}\right), \quad y \in B\left(x_{1}, \frac{r}{8}\right),
$$

for some constant $c_{2}>0$. Therefore, by (2.7) and (2.11)-(2.12),

$$
u\left(x_{2}\right)=\mathbb{E}_{x_{2}}\left[u\left(X_{\tau_{B\left(x_{2}, \frac{r}{8}\right)}}\right)\right] \geq \mathbb{E}_{x_{2}}\left[u\left(X_{\left.\tau_{B\left(x_{2}, \frac{r}{8}\right)}\right)}\right) ; X_{\tau_{B\left(x_{2}, \frac{r}{8}\right)}} \in B\left(x_{1}, \frac{r}{8}\right)\right]
$$




$$
\begin{aligned}
& \geq c_{2} u\left(x_{1}\right) \mathbb{P}_{x_{2}}\left(X_{\tau_{B\left(x_{2}, \frac{r}{8}\right)}} \in B\left(x_{1}, \frac{r}{8}\right)\right)=c_{2} u\left(x_{1}\right) \int_{B\left(x_{1}, \frac{r}{8}\right)} K_{B\left(x_{2}, \frac{r}{8}\right)}\left(x_{2}, w\right) d w \\
& \geq c_{3} u\left(x_{1}\right)\left|B\left(x_{1}, \frac{r}{8}\right)\right| r^{-d}=c_{4} u\left(x_{1}\right) .
\end{aligned}
$$

Thus we have proved the right-hand side inequality in the conclusion of the theorem. The inequality on the left-hand side follows by symmetry.

For notational convenience, we define

$$
\Phi(r)=\frac{1}{\phi\left(r^{-2}\right)}, \quad r>0 .
$$

The inverse function of $\Phi$ will be denoted by the usual notation $\Phi^{-1}(r)$.

Our process $X$ belongs to the class of Markov processes considered in [7]. Thus we have the following two-sided estimates for $p(t, x, y)$ from [7]. The proof is the same as that of [9, Proposition $2.2]$.

Proposition 2.11 For any $T>0$, there exists $c_{1}=c_{1}\left(T, \Psi, \gamma_{1}, \gamma_{2}\right)>0$ such that

$$
p(t, x, y) \leq c_{1}\left(\Phi^{-1}(t)\right)^{-d} \quad \text { for all }(t, x, y) \in(0, T] \times \mathbb{R}^{d} \times \mathbb{R}^{d} .
$$

For any $T, R>0$, there exists $c_{2}=c_{2}\left(T, R, \Psi, \gamma_{1}, \gamma_{2}\right)>1$ such that for all $(t, x, y) \in[0, T] \times \mathbb{R}^{d} \times \mathbb{R}^{d}$ with $|x-y|<R$,

$$
c_{2}^{-1}\left(\left(\Phi^{-1}(t)\right)^{-d} \wedge \frac{t}{|x-y|^{d} \Phi(|x-y|)}\right) \leq p(t, x, y) \leq c_{2}\left(\left(\Phi^{-1}(t)\right)^{-d} \wedge \frac{t}{|x-y|^{d} \Phi(|x-y|)}\right) .
$$

Our argument to obtain upper bound on the Green functions of bounded open sets is similar to those in [8, 15]. We give the details here for the completeness.

Lemma 2.12 For every bounded open set $D$, the Green function $G_{D}(x, y)$ is finite and continuous off the diagonal of $D \times D$ and there exists $c=c\left(\operatorname{diam}(D), \Psi, \gamma_{1}, \gamma_{2}\right) \geq 1$ such that for all $x, y \in D$,

$$
G_{D}(x, y) \leq c \frac{\Phi(|x-y|)}{|x-y|^{d}}=\frac{c}{|x-y|^{d} \phi\left(|x-y|^{-2}\right)}
$$

Proof. Put $L:=\operatorname{diam}(D)$. By (2.15), for every $x \in D$ we have

$$
\begin{aligned}
& \mathbb{P}_{x}\left(\tau_{D} \leq 1\right) \geq \mathbb{P}_{x}\left(X_{1} \in \mathbb{R}^{d} \backslash D\right)=\int_{\mathbb{R}^{d} \backslash D} p(1, x, y) d y \\
& \geq c_{1} \int_{\mathbb{R}^{d} \backslash D}\left(1 \wedge \frac{1}{|x-y|^{d} \Phi(|x-y|)}\right) d y \geq c_{1} \int_{\{|z| \geq L\}}\left(1 \wedge \frac{1}{|z|^{d} \Phi(|z|)}\right) d z=c_{2}>0 .
\end{aligned}
$$

Thus

$$
\sup _{x \in D} \int_{D} p_{D}(1, x, y) d y=\sup _{x \in D} \mathbb{P}_{x}\left(\tau_{D}>1\right)<1 .
$$


Now the Markov property of $X$ implies that there exist positive constants $c_{3}$ and $c_{4}$ such that

$$
\int_{D} p_{D}(t, x, y) d y \leq c_{3} e^{-c_{4} t} \text { for all }(t, x) \in(0, \infty) \times D .
$$

Thus combining this, (2.15) and the semigroup property, we have that for any $(t, x, y) \in(1, \infty) \times$ $D \times D$,

$$
p_{D}(t, x, y)=\int_{D} p_{D}(t-1, x, z) p_{D}(1, z, y) d z \leq c_{5} \int_{D} p_{D}(t-1, x, z) d z \leq c_{6} e^{-c_{4} t} .
$$

This with (2.15) implies that such that for any $(x, y) \in D \times D$,

$$
G_{D}(x, y)=\int_{0}^{\infty} p_{D}(t, x, y) d t \leq c_{7} \int_{0}^{1}\left(\left(\Phi^{-1}(t)\right)^{-d} \wedge \frac{t}{|x-y|^{d} \Phi(|x-y|)}\right) d t+c_{7} .
$$

By the proof of [7, Theorem 6.1],

$$
\int_{0}^{1}\left(\left(\Phi^{-1}(t)\right)^{-d} \wedge \frac{t}{|x-y|^{d} \Phi(|x-y|)}\right) d t \asymp \frac{\Phi(|x-y|)}{|x-y|^{d}} .
$$

Therefore the Green function $G_{D}(x, y)=\int_{0}^{\infty} p_{D}(t, x, y) d t$ is finite and continuous off the diagonal of $D \times D$. Furthermore, by (2.3), we have $\inf _{a \leq \operatorname{diam}(D)} \Phi(a) a^{-d}>0$. Consequently, (2.16) holds.

For interior lower bound on the Green function, we use some recent results from [9]. The next result is an analog of [9, Proposition 3.6], which is the main result of [9, Section 3]. Even though it is assumed in [9] that $X$ is rotationally symmetric and its Lévy density satisfies a little stronger assumption than in this paper, all the arguments of [9, Section 3] only use the results in [7], (1.1), (1.2), (2.2), (2.3), and the semigroup property. Thus by following the same arguments line by line, one can prove the next proposition. We omit the details. We note in passing that $D$ is not necessarily bounded in the next proposition.

Proposition 2.13 Let $T$ and $a$ be positive constants. There exists $c=c\left(T, a, \Psi, \gamma_{1}, \gamma_{2}\right)>0$ such that for any open set $D$,

$$
p_{D}(t, x, y) \geq c\left(\left(\Phi^{-1}(t)\right)^{-d} \wedge t j(|x-y|)\right)
$$

for every $(t, x, y) \in(0, T] \times D \times D$ with $\delta_{D}(x) \wedge \delta_{D}(y) \geq a \Phi^{-1}(t)$.

Lemma 2.14 For every $L, T>0$, there exists $c=c\left(T, L, \Psi, \gamma_{1}, \gamma_{2}\right)>0$ such that for any bounded open set $D$ with $\operatorname{diam}(D) \leq T, x, y \in D$ with $|x-y| \leq L\left(\delta_{D}(x) \wedge \delta_{D}(y)\right)$,

$$
G_{D}(x, y) \geq c \frac{\Phi(|x-y|)}{|x-y|^{d}}=\frac{c}{|x-y|^{d} \phi\left(|x-y|^{-2}\right)} .
$$


Proof. Without loss of generality, we assume $L \geq 1, \delta_{D}(y) \leq \delta_{D}(x)$ and $\operatorname{diam}(D) \leq T$. By Proposition 2.13 and (2.2), there exists $c_{1}=c_{1}\left(T, \Psi, \gamma_{1}, \gamma_{2}\right)>0$ such that for all $(t, z, w) \in$ $(0, T] \times D \times D$ with $\delta_{D}(z) \wedge \delta_{D}(w) \geq \Phi^{-1}(t)$,

$$
p_{D}(t, z, w) \geq c_{1}\left(\left(\Phi^{-1}(t)\right)^{-d} \wedge \frac{t}{|z-w|^{d} \Phi(|z-w|)}\right) .
$$

Using this we have

$$
\begin{aligned}
& G_{D}(x, y) \geq \int_{0}^{T} p_{D}(t, x, y) d t \geq \int_{0}^{\Phi\left(\delta_{D}(y)\right)} p_{D}(t, x, y) d t \\
& \geq c_{1} \int_{0}^{\Phi\left(\delta_{D}(y)\right)}\left(\Phi^{-1}(t)\right)^{-d} \wedge \frac{t}{|x-y|^{d} \Phi(|x-y|)} d t \\
& \geq c_{1} \int_{0}^{\Phi\left(L^{-1}|x-y|\right)}\left(\Phi^{-1}(t)\right)^{-d} \wedge \frac{t}{|x-y|^{d} \Phi(|x-y|)} d t .
\end{aligned}
$$

Let $r=|x-y|$. By the change of variable $u=\frac{\Phi(r)}{t}$ and the fact that $t \rightarrow \Phi(t)$ is increasing,

$$
\begin{aligned}
\int_{0}^{\Phi\left(L^{-1} r\right)}\left(\Phi^{-1}(t)\right)^{-d} \wedge \frac{t}{r^{d} \Phi(r)} d t & =\frac{\Phi(r)}{r^{d}} \int_{\Phi(r) / \Phi\left(L^{-1} r\right)}^{\infty} u^{-2}\left(\left(\frac{r}{\Phi^{-1}\left(u^{-1} \Phi(r)\right)}\right)^{d} \wedge u^{-1}\right) d u \\
& =\frac{\Phi(r)}{r^{d}} \int_{\Phi(r) / \Phi\left(L^{-1} r\right)}^{\infty} u^{-3} d u
\end{aligned}
$$

Since by (2.3) and (2.13)

$$
\int_{\Phi(r) / \Phi\left(L^{-1} r\right)}^{\infty} u^{-3} d u \geq \int_{c_{2} L^{2 \delta_{1}}}^{\infty} u^{-3} d u>0
$$

we conclude from (2.20) and (2.21) that (2.18) holds.

It follows from (2.3), Lemmas 2.14 and 2.5 that, for all $r \in(0,1)$ and all $\alpha \in(0, \pi)$, there exists $c=c(\alpha)>0$ such that for all cones $V$ of angle $\alpha$ with vertex at the origin,

$$
\begin{aligned}
& \mathbb{E}_{0} \int_{0}^{\tau_{B(0, r)}} 1_{V}\left(X_{s}\right) d s \geq \int_{V \cap B(0, r / 2)} G_{B(0, r)}(0, y) d y \geq c_{1} \int_{V \cap B(0, r / 2)} \frac{1}{|y|^{d} \phi\left(|y|^{-2}\right)} d y \\
& \geq c_{2} \int_{0}^{r / 2} \frac{1}{r \phi\left(r^{-2}\right)} d r \geq c_{3} \phi\left(r^{-2}\right) \geq c_{4} \mathbb{E}_{0} \tau_{B(0, r)} .
\end{aligned}
$$

Thus $X$ satisfies hypothesis $\mathbf{H}$ in [30]. It follows from (1.2), (2.2) and Lemma 2.4 that for every cone $V$ with vertex at the origin,

$$
\int_{V \cap B(0,1)} \mathbb{E}_{0} \tau_{B(0,|y|)} J_{X}(y) d y \geq c \int_{V \cap B(0,1)}|y|^{-d} d y=\infty .
$$

Therefore it follows from [30, Theorem 1] that if $V$ is a Lipschitz open set in $\mathbb{R}^{d}$ and $D$ is an open subset of $V$,

$$
\mathbb{P}_{x}\left(X_{\tau_{D}} \in \partial V\right)=0, \quad x \in D
$$


Using Theorem 2.8, the proof of the next result is the same as that of [18, Proposition 13.4.11]. So we omit the proof.

Proposition 2.15 For every $a \in(0,1)$, there exists $c=c\left(\Psi, \gamma_{1}, \gamma_{2}, a\right)>0$ such that for every $r \in(0,1], x_{0} \in \mathbb{R}^{d}$ and $x_{1}, x_{2} \in B\left(x_{0}, a r\right)$,

$$
K_{B\left(x_{0}, r\right)}\left(x_{1}, y\right) \leq c K_{B\left(x_{0}, r\right)}\left(x_{2}, y\right), \quad \text { a.e. } y \in{\overline{B\left(x_{0}, r\right)}}^{c} .
$$

Proposition 2.16 For every $a \in(0,1)$, there exists $c=c\left(\Psi, \gamma_{1}, \gamma_{2}, a\right)>0$ such that for every $r \in(0,1]$ and $x_{0} \in \mathbb{R}^{d}$,

$$
K_{B\left(x_{0}, r\right)}(x, y) \leq c r^{-d}\left(\frac{\phi\left(\left(\left|y-x_{0}\right|-r\right)^{-2}\right)}{\phi\left(r^{-2}\right)}\right)^{1 / 2}
$$

for all $x \in B\left(x_{0}\right.$, ar $)$ and a.e. $y$ such that $r<\left|x_{0}-y\right|<2 r$.

Proof. Using (2.2), Lemmas 2.2, 2.5 and Proposition 2.15, the proof is exactly the same as that of [21, Proposition 4.9]. We omit it.

Lemma 2.17 For every $a \in(0,1)$, there exists a constant $c=c\left(\Psi, \gamma_{1}, \gamma_{2}, a\right)>0$ such that for any $r \in(0,1)$ and any open set $D$ with $D \subset B(0, r)$ we have

$$
\mathbb{P}_{x}\left(X_{\tau_{D}} \in B(0, r)^{c}\right) \leq c \phi\left(r^{-2}\right) \int_{D} G_{D}(x, y) d y, \quad x \in D \cap B(0, a r) .
$$

Proof. Using Lemma 2.3, the proof is exactly the same as that of [21, Lemma 4.10]. We omit it.

With these preparations in hand, we can repeat the argument of [21, Section 5] to get the following form of the boundary Harnack principle established in [21]. We omit the details. Note that the open set $D$ in the next result is not necessarily bounded.

Theorem 2.18 There exists $C_{1}=C_{1}\left(\Psi, \gamma_{1}, \gamma_{2}\right) \geq 1$ such that the following hold for all $r \in(0,1)$.

(i) For every $z_{0} \in \mathbb{R}^{d}$, every open set $U \subset B\left(z_{0}, r\right)$ and for any nonnegative function $u$ in $\mathbb{R}^{d}$ which is regular harmonic in $U$ with respect to $X$ and vanishes a.e. in $U^{c} \cap B\left(z_{0}, r\right)$ it holds that

$$
C_{1}^{-1} \mathbb{E}_{x}\left[\tau_{U}\right] \int_{B\left(z_{0}, r / 2\right)^{c}} j\left(\left|y-z_{0}\right|\right) u(y) d y \leq u(x) \leq C_{1} \mathbb{E}_{x}\left[\tau_{U}\right] \int_{B\left(z_{0}, r / 2\right)^{c}} j\left(\left|y-z_{0}\right|\right) u(y) d y
$$

for every $x \in U \cap B\left(z_{0}, r / 2\right)$. 
(ii) For every $z_{0} \in \mathbb{R}^{d}$, every open set $D \subset \mathbb{R}^{d}$, every $r \in(0,1)$ and for any nonnegative functions $u, v$ in $\mathbb{R}^{d}$ which are regular harmonic in $D \cap B\left(z_{0}, r\right)$ with respect to $X$ and vanish a.e. in $D^{c} \cap B\left(z_{0}, r\right)$, we have

$$
\frac{u(x)}{v(x)} \leq C_{1}^{4} \frac{u(y)}{v(y)}, \quad x, y \in D \cap B\left(z_{0}, r / 2\right)
$$

(iii) For every $z_{0} \in \mathbb{R}^{d}$, every Greenian open set $D \subset \mathbb{R}^{d}$, every $r \in(0,1)$, we have

$$
K_{D}\left(x_{1}, y_{1}\right) K_{D}\left(x_{2}, y_{2}\right) \leq C_{1}^{4} K_{D}\left(x_{1}, y_{2}\right) K_{D}\left(x_{2}, y_{1}\right)
$$

for all $x_{1}, x_{2} \in D \cap B\left(z_{0}, r / 2\right)$ and a.e. $y_{1}, y_{2} \in \bar{D}^{c} \cap B\left(z_{0}, r\right)^{c}$.

In the next two results, we will assume that $X$ is transient and will use $G(x, y)$ to denote the Green function of $X$. Note that $G(x, y)=G(y, x)$ by symmetry and $G(x, y)=G(0, y-x)$ by translation invariance. Since we only assume that $X$ is symmetric, rather than unimodal, the next result does not follow from [13].

Theorem 2.19 For every $M \geq 1$ there exists a constant $C_{2}(M)=C_{2}\left(M, \Psi, \gamma_{1}, \gamma_{2}\right)>0$ such that for all $x \in B(0, M)$,

$$
C_{2}(M)^{-1} \frac{\Phi(|x|)}{|x|^{d}} \leq G(x, 0) \leq C_{2}(M) \frac{\Phi(|x|)}{|x|^{d}}
$$

Proof. In this proof, we always assume that $x \in B(0, M)$. It follows from Lemma 2.14 that

$$
G(x, 0) \geq G_{B(0,2 M)}(x, 0) \geq c_{1} \frac{\Phi(|x|)}{|x|^{d}} .
$$

On the other hand, by the strong Markov property and Lemma 2.12 ,

$$
G(x, 0)=G_{B(0,2 M)}(x, 0)+\mathbb{E}_{x}\left[G\left(X_{\tau_{B(0,2 M)}}, 0\right)\right] \leq c_{2} \frac{\Phi(|x|)}{|x|^{d}}+\mathbb{E}_{x}\left[G\left(X_{\tau_{B(0,2 M)}}, 0\right)\right] .
$$

Choose $x_{0}=(0, \ldots, 0, M / 2)$. By Theorem 2.10 and the strong Markov property, we have

$$
\mathbb{E}_{x}\left[G\left(X_{\tau_{B(0,2 M)}}, 0\right)\right] \leq c_{3} \mathbb{E}_{x_{0}}\left[G\left(X_{\tau_{B(0,2 M)}}, 0\right)\right] \leq c_{3} G\left(x_{0}, 0\right) \leq c_{4}<\infty
$$

Furthermore, by (2.3), we have $\inf _{a \leq 2 M} \Phi(a) a^{-d}>0$. Thus we conclude that

$$
G(x, 0)=G_{B(0,2 M)}(x, 0)+\mathbb{E}_{x}\left[G\left(X_{\tau_{B(0,2 M)}}, 0\right)\right] \leq c_{2} \frac{\Phi(|x|)}{|x|^{d}}+c_{4} \leq c_{5} \frac{\Phi(|x|)}{|x|^{d}} .
$$

The following result will be needed in the proof of Theorem 3.12 . 
Lemma 2.20 If $X$ is transient, then

$$
\lim _{x \rightarrow \infty} G(x, 0)=0
$$

Proof. It follows from the transience assumption and (1.5) that $1 / \Psi_{X}$ is locally integrable in $\mathbb{R}^{d}$, thus by [1, Proposition 13.23] that the semigroup of $X$ is integrable (in the sense of [1]). Therefore it follows from [1, Proposition 13.21] that, for any nonnegative continuous function $f$ on $\mathbb{R}^{d}$ with compact support, $\lim _{x \rightarrow \infty} G f(x)=0$.

By Theorem 2.8, for any $x \in B(0,4)^{c}$,

$$
G(x, 0) \leq c G(x, y), \quad y \in B(0,2) .
$$

Take a nonnegative function $f$ with support in $\overline{B(0,2)}$ such that $f$ is identically 1 on $B(0,1)$. Then

$$
G(x, 0) \int_{B(0,2)} f(y) d y=\int_{B(0,2)} G(x, 0) f(y) d y \leq c \int_{B(0,2)} G(x, y) f(y) d y=G f(x)
$$

which yields

$$
G(x, 0) \leq c \frac{G f(x)}{\int_{B(0,2)} f(y) d y} .
$$

Therefore $\lim _{x \rightarrow \infty} G(x, 0)=0$.

Remark 2.21 Note that several results of this section are stated only for small radii $r$, namely $r \in(0,1]$. This is, of course, a consequence of the scaling condition $(\mathbf{H})$ at infinity which governs the behavior of the process for small time and small space. If we want to study the large time and large space behavior of $X$, we would need to add the following scaling condition on $\Psi$ near the origin too:

(H2): There exist constants $0<\delta_{3} \leq \delta_{4}<1$ and $a_{3}, a_{4}>0$ such that

$$
a_{3} \lambda^{2 \delta_{4}} \Psi(t) \leq \Psi(\lambda t) \leq a_{4} \lambda^{2 \delta_{3}} \phi(t), \quad \lambda \leq 1, t \leq 1
$$

One consequence of adding condition (H2) to condition $(\mathbf{H})$ is that many results that were valid for small $r$ only will hold true for all $r>0$. For future reference, we list below precisely which statements are true.

First note that if both $(\mathbf{H})$ and $(\mathbf{H 2})$ hold, there exist $a_{5}, a_{6}>0$ such that

$$
a_{5}\left(\frac{R}{r}\right)^{2\left(\delta_{1} \wedge \delta_{3}\right)} \leq \frac{\Psi(R)}{\Psi(r)} \leq a_{6}\left(\frac{R}{r}\right)^{2\left(\delta_{2} \vee \delta_{4}\right)}, \quad a>0,0<r<R<\infty
$$

cf. [22, (2.6)].

In the remainder of this remark, in addition to all conditions from Section 1, namely (1.1), (1.2) and (H), we also assume $(\mathbf{H 2})$. Then $X$ satisfies the assumptions in [10]. Furthermore, it follows 
from [4, (15), Corollary 23, Proposition 28] and (2.23) that (2.2) and (2.4) hold for all $r>0$. That is, under both $(\mathbf{H})$ and $(\mathbf{H 2})$

$$
j(r) \asymp \frac{\phi\left(r^{-2}\right)}{r^{d}} \quad \text { for } r>0,
$$

and

$$
j(r) \leq c j(2 r), \quad r>0 .
$$

The following results are now true for all $r>0$ : Theorem 2.8, Lemma 2.9, Theorem 2.10, Proposition 2.15, Proposition 2.16, Lemma 2.17 and Theorem 2.18, In order to prove Theorem 2.8, we also have to use [10, Theorem 4.12], while in the proof of Theorem 2.10 we use (2.24) instead of (2.2). Furthermore, using results in [10, Proposition 2.13 and Lemma 2.14 hold with $T=\infty$. Proofs of all other listed results stay the same.

\section{Martin Boundary}

In this section we will use the boundary Harnack principle (Theorem 2.18) and follow the wellestablished route, see [2, 17, 19, 23], to study the Martin boundary of an open set $D$ with respect to $X$. The key ingredient is the oscillation reduction technique used in the proof of Lemma 3.4.

Throughout this section we assume that $D \subset \mathbb{R}^{d}$ is a Greenian open set, $Q \in \bar{D}$ and $D$ is $\kappa$-fat at $Q$ for some $\kappa \in\left(0, \frac{1}{2}\right)$, that is, there is $R>0$ such that for all $r \in(0, R]$, there is a ball $B\left(A_{r}(Q), \kappa r\right) \subset D \cap B(Q, r)$. Without loss of generality, we assume that $R \leq 1 / 2$. After Lemma 3.4, we will further assume that $Q \in \partial D$.

Lemma 3.1 There exist $C_{3}=C_{3}\left(\Psi, \kappa, \gamma_{1}, \gamma_{2}\right)>0$ and $\xi=\xi\left(\Psi, \kappa, \gamma_{1}, \gamma_{2}\right) \in(0,1)$ such that for every $r \in(0, R)$ and any non-negative function $h$ in $\mathbb{R}^{d}$ which is harmonic in $D \cap B(Q, r)$ it holds that

$$
h\left(A_{r}(Q)\right) \leq C_{3}\left(\phi\left(r^{-2}\right)\right)^{-1} \xi^{k} \phi\left((\kappa / 2)^{-2 k} r^{-2}\right) h\left(A_{(\kappa / 2)^{k} r}(Q)\right), \quad k=0,1,2, \ldots
$$

Proof. Without loss of generality, we may assume that $Q=0$. Fix $r \in(0, R)$. For $k=0,1,2, \ldots$, let $\eta_{k}=(\kappa / 2)^{k} r, A_{k}=A_{\eta_{k}}(0)$ and $B_{k}=B\left(A_{k}, \eta_{k+1}\right)$. Note that the balls $B_{k}$ are pairwise disjoint. By harmonicity of $h$, for every $k=0,1,2 \ldots$,

$$
h\left(A_{k}\right)=\mathbb{E}_{A_{k}}\left[h\left(X_{\tau_{B_{k}}}\right)\right] \geq \sum_{l=0}^{k-1} \mathbb{E}_{A_{k}}\left[h\left(X_{\tau_{B_{k}}}\right): X_{\tau_{B_{k}}} \in B_{l}\right]=\sum_{l=0}^{k-1} \int_{B_{l}} K_{B_{k}}\left(A_{k}, z\right) h(z) d z .
$$

By Theorem [2.10, there exists $c_{1}=c_{1}\left(\Psi, \gamma_{1}, \gamma_{2}\right)>0$ such that for every $l=0,1,2, \ldots, h(z) \geq$ $c_{1} h\left(A_{l}\right)$ for all $z \in B_{l}$. Hence

$$
\int_{B_{l}} K_{B_{k}}\left(A_{k}, z\right) h(z) d z \geq c_{1} h\left(A_{l}\right) \int_{B_{l}} K_{B_{k}}\left(A_{k}, z\right) d z, \quad 0 \leq l \leq k-1 .
$$


By (2.10), we have

$$
\int_{B_{l}} K_{B_{k}}\left(A_{k}, z\right) d z \geq c_{2} \phi\left(\eta_{k}^{-2}\right)^{-1} \int_{B_{l}} j\left(\left|2\left(A_{k}-z\right)\right|\right) d z, \quad 0 \leq l \leq k-1 .
$$

For $l=0,1, \cdots, k-1$ and $z \in B_{l}$, it holds that $|z| \leq \kappa(\kappa / 2)^{l} r+(\kappa / 2)^{(l+1)} r=(3 \kappa / 2)(\kappa / 2)^{l} r$. Since $\left|A_{k}\right| \leq \kappa \eta_{k}$, we have that $\left|A_{k}-z\right| \leq\left|A_{k}\right|+|z|<3 \eta_{l}$. Together with (2.2) and Lemma 2.1, this implies that $j\left(\left|2\left(A_{k}-z\right)\right|\right) \geq c_{3}\left|\eta_{l}\right|^{-d} \phi\left(\eta_{k}^{-2}\right)$ for every $z \in B_{l}$ and $0 \leq l \leq k-1$. Therefore,

$$
\int_{B_{l}} K_{B_{k}}\left(A_{k}, z\right) d z \geq c_{4}\left|\eta_{l}\right|^{-d} \phi\left(\eta_{k}^{-2}\right) \phi\left(\eta_{k}^{-2}\right)^{-1}\left|B_{l}\right| \geq c_{5} \phi\left(\eta_{l}^{-2}\right) / \phi\left(\eta_{k}^{-2}\right), \quad 0 \leq l \leq k-1 .
$$

Hence,

$$
\phi\left(\eta_{k}^{-2}\right) h\left(A_{k}\right) \geq c_{5} \sum_{l=0}^{k-1} \phi\left(\eta_{l}^{-2}\right) h\left(A_{l}\right), \quad \text { for all } k=1,2, \ldots,
$$

where $c_{5}=c_{5}\left(\Psi, \gamma_{1}, \gamma_{2}, \kappa\right)$. Let $a_{k}:=\phi\left(\eta_{k}^{-2}\right) h\left(A_{k}\right)$ so that $a_{k} \geq c_{5} \sum_{l=0}^{k-1} a_{l}$. Using the identity $1+c_{5} \sum_{l=0}^{k-2}\left(1+c_{5}\right)^{l}=\left(1+c_{5}\right)^{k-1}$ for $k \geq 3$, by induction it follows that $a_{k} \geq c_{5}\left(1+c_{5}\right)^{k-1} a_{0}$. Let $\xi:=\left(1+c_{5}\right)^{-1} \in(0,1)$ so that

$$
\phi\left(r^{-2}\right) h\left(A_{0}\right)=a_{0} \leq\left(1+c_{5}\right) c_{5}^{-1} \xi^{k} a_{k}=\left(1+c_{5}\right) c_{5}^{-1} \xi^{k} \phi\left(\eta_{k}^{-2}\right) h\left(A_{k}\right) .
$$

Then,

$$
h\left(A_{r}(0)\right) \leq\left(1+c_{5}\right) c_{5}^{-1}\left(\phi\left(r^{-2}\right)\right)^{-1} \xi^{k} \phi\left(\eta_{k}^{-2}\right) h\left(A_{(\kappa / 2)^{k} r}(0)\right) .
$$

Lemma 3.2 There exists $c=c\left(\Psi, \kappa, \gamma_{1}, \gamma_{2}\right)>0$ such that for every $r \in(0, R)$ and every nonnegative function $u$ in $\mathbb{R}^{d}$ which is regular harmonic in $D \cap B(Q, r)$ with respect to $X$,

$$
u\left(A_{r}(Q)\right) \geq c \phi\left(r^{-2}\right)^{-1} \int_{B(Q, r)^{c}} j(|z-Q|) u(z) d z .
$$

Proof. Without loss of generality, we may assume that $Q=0$. Fix $r \in(0, R)$ and let $A:=A_{r}(0)$. Since $u$ is regular harmonic in $D \cap B(0,(1-\kappa / 2) r)$ with respect to $X$, we have

$$
\begin{aligned}
u(A) & \geq \mathbb{E}_{A}\left[u\left(X_{\tau_{D \cap B(0,(1-\kappa / 2) r)}}\right): X_{\tau_{D \cap B(0,(1-\kappa / 2) r)}} \in B(0, r)^{c}\right] \\
& =\int_{B(0, r)^{c}} K_{D \cap B(0,(1-\kappa / 2) r)}(A, z) u(z) d z \\
& =\int_{B(0, r)^{c}} \int_{D \cap B(0,(1-\kappa / 2) r)} G_{D \cap B(0,(1-\kappa / 2) r)}(A, y) J_{X}(y-z) d y u(z) d z .
\end{aligned}
$$

Since $B(A, \kappa r / 2) \subset D \cap B(0,(1-\kappa / 2) r)$, by the domain monotonicity of Green functions,

$$
G_{D \cap B(0,(1-\kappa / 2) r)}(A, y) \geq G_{B(A, \kappa r / 2)}(A, y), \quad y \in B(A, \kappa r / 2) .
$$


Thus

$$
\begin{aligned}
u(A) & \geq \int_{B(0, r)^{c}} \int_{B(A, \kappa r / 2)} G_{B(A, \kappa r / 2)}(A, y) J_{X}(y-z) d y u(z) d z \\
& =\int_{B(0, r)^{c}} K_{B(A, \kappa r / 2)}(A, z) u(z) d z \\
& \geq c_{1} \phi\left((\kappa r / 2)^{-2}\right)^{-1} \int_{B(0, r)^{c}} j(|A-z|) u(z) d z,
\end{aligned}
$$

where in the last line we used (2.10). Note that $|A-z| \leq 2|z|$ for $z \in A(0, r, 1)$ and $|A-z| \leq|z|+1$ for $z \in B(0,1)^{c}$. Hence by (1.1), (2.4) and Lemma 2.1,

$$
u(A) \geq c_{2} \phi\left(r^{-2}\right)^{-1} \int_{B(0, r)^{c}} j(|z|) u(z) d z .
$$

Lemma 3.3 There exist $C_{4}=C_{4}\left(\Psi, \kappa, \gamma_{1}, \gamma_{2}\right) \geq 1$ and $\xi=\xi\left(\Psi, \kappa, \gamma_{1}, \gamma_{2}\right) \in(0,1)$ such that for any $r \in(0,1)$, and any non-negative function $u$ on $\mathbb{R}^{d}$ which is regular harmonic in $D \cap B(Q, r)$ with respect to $X$ and vanishes in $D^{c} \cap B(Q, r)$ we have

$$
\mathbb{E}_{x}\left[u\left(X_{\tau_{D \cap B_{k}}}\right): X_{\tau_{D \cap B_{k}}} \in B(Q, r)^{c}\right] \leq C_{4} \xi^{k} u(x), \quad x \in D \cap B_{k},
$$

where $B_{k}:=B\left(Q,(\kappa / 2)^{k} r\right), k=0,1,2, \cdots$.

Proof. Without loss of generality, we may assume that $Q=0$. Fix $r \in(0, R)$. Let $\eta_{k}:=(\kappa / 2)^{k} r$, $B_{k}:=B\left(0, \eta_{k}\right)$ and

$$
u_{k}(x):=\mathbb{E}_{x}\left[u\left(X_{\tau_{D \cap B_{k}}}\right): X_{\tau_{D \cap B_{k}}} \in B(0, r)^{c}\right], \quad x \in D \cap B_{k} .
$$

Note that for $x \in D \cap B_{k+1}$,

$$
\begin{aligned}
u_{k+1}(x) & =\mathbb{E}_{x}\left[u\left(X_{\tau_{D \cap B_{k+1}}}\right): X_{\tau_{D \cap B_{k+1}}} \in B(0, r)^{c}\right] \\
& =\mathbb{E}_{x}\left[u\left(X_{\tau_{D \cap B_{k+1}}}\right): \tau_{D \cap B_{k+1}}=\tau_{D \cap B_{k}}, X_{\tau_{D \cap B_{k+1}}} \in B(0, r)^{c}\right] \\
& =\mathbb{E}_{x}\left[u\left(X_{\tau_{D \cap B_{k}}}\right): \tau_{D \cap B_{k+1}}=\tau_{D \cap B_{k}}, X_{\tau_{D \cap B_{k}}} \in B(0, r)^{c}\right] \\
& \leq \mathbb{E}_{x}\left[u\left(X_{\tau_{D \cap B_{k}}}\right): X_{\tau_{D \cap B_{k}}} \in B(0, r)^{c}\right] .
\end{aligned}
$$

Thus

$$
u_{k+1}(x) \leq u_{k}(x), \quad x \in D \cap B_{k+1} .
$$

Let $A_{k}:=A_{\eta_{k}}(0)$. Similarly we have

$$
u_{k}\left(A_{k}\right)=\mathbb{E}_{A_{k}}\left[u\left(X_{\tau_{D \cap B_{k}}}\right): X_{\tau_{D \cap B_{k}}} \in B(0, r)^{c}\right]
$$




$$
\begin{aligned}
& \leq \mathbb{E}_{A_{k}}\left[u\left(X_{\tau_{B_{k}}}\right): X_{\tau_{D \cap B_{k}}} \in B(0, r)^{c}\right] \\
& \leq \int_{B(0, r)^{c}} K_{B_{k}}\left(A_{k}, z\right) u(z) d z .
\end{aligned}
$$

By (2.9), we have

$$
K_{B_{k}}\left(A_{k}, z\right) \leq c_{1} j\left(|z|-\eta_{k}\right) \phi\left(\eta_{k}^{-2}\right)^{-1}, \quad z \in B(0, r)^{c} .
$$

Note that $|z|-\eta_{k} \geq|z| / 2$ for $z \in A(0, r, 2)$ and $|z|-\eta_{k} \geq|z|-1$ for $z \geq 2$. Thus by (1.1), (2.4) and the monotonicity of $j$,

$$
u_{k}\left(A_{k}\right) \leq c_{2} \phi\left(\eta_{k}^{-2}\right)^{-1} \int_{B(0, r)^{c}} j(|z|) u(z) d z, \quad k=1,2, \cdots
$$

By Lemma 3.2 , we have

$$
u\left(A_{0}\right) \geq c_{3} \phi\left(\eta_{1}^{-2}\right)^{-1} \int_{B(0, r)^{c}} j(|z|) u(z) d z .
$$

Thus (3.3)-(3.4) imply that

$$
u_{k}\left(A_{k}\right) \leq c_{4} \phi\left(\eta_{1}^{-2}\right) / \phi\left(\eta_{k}^{-2}\right) u\left(A_{0}\right) .
$$

On the other hand, using Lemma 3.1, we get

$$
u\left(A_{0}\right) \leq c_{5}\left(\phi\left(r^{-2}\right)\right)^{-1} \xi^{k} \phi\left(\eta_{k}^{-2}\right) u\left(A_{k}\right) .
$$

Combining the last two displays and using Lemma 2.1, we get

$$
u_{k}\left(A_{k}\right) \leq c_{6} \xi^{k} \frac{\phi\left(\eta_{1}^{-2}\right)}{\phi\left(r^{-2}\right)} u\left(A_{k}\right) \leq c_{7} \xi^{k} u\left(A_{k}\right) .
$$

By the boundary Harnack principle, Theorem 2.18 (i), we have

$$
\frac{u_{k}(x)}{u(x)} \leq \frac{u_{k-1}(x)}{u(x)} \leq c_{8} \frac{u_{k-1}\left(A_{k-1}\right)}{u\left(A_{k-1}\right)} \leq c_{9} \xi^{k}
$$

for $k=1,2, \cdots$. The proof is now complete.

Using the boundary Harnack principle and Lemma 3.3 (instead of Lemmas 13 and 14 in [2]), we can repeat the argument of [2, Lemma 16] (which dealt with isotropic stable process) to get the following result. We include the proof from [2] to show that it does not depend on scaling property of stable processes, and on the way make some constants explicit and provide the detailed computation in the end of the induction argument.

Lemma 3.4 There exist $c=c\left(\Psi, \gamma_{1}, \gamma_{2}, \kappa\right)>0$ and $\beta=\beta\left(\Psi, \gamma_{1}, \gamma_{2}, \kappa\right)>0$ such that for all $r \in(0, R / 2)$ and non-negative functions $u$ and $v$ on $\mathbb{R}^{d}$ which are regular harmonic in $D \cap B(Q, 2 r)$ with respect to $X$, vanish on $D^{c} \cap B(Q, 2 r)$ and satisfy $u\left(A_{r}(Q)\right)=v\left(A_{r}(Q)\right)$, the limit $g=$ $\lim _{D \ni x \rightarrow Q} \frac{u(x)}{v(x)}$ exists, and we have

$$
\left|\frac{u(x)}{v(x)}-g\right| \leq c\left(\frac{|x-Q|}{r}\right)^{\beta}, \quad x \in D \cap B(Q, r) .
$$


Proof. Fix $r \in(0, R / 2)$. Without loss of generality, we assume that $u\left(A_{r}(Q)\right)=v\left(A_{r}(Q)\right)=1$. Let $D_{0}=D \cap B(Q, r)$. We start the proof by fixing several constants. We first choose $c_{1}=$ $c_{1}\left(\Psi, \gamma_{1}, \gamma_{2}\right) \geq 10$ such that

$$
\sup _{x \in D_{0}} \frac{u(x)}{v(x)} \leq\left(1+\frac{c_{1}}{2}\right) \inf _{x \in D_{0}} \frac{u(x)}{v(x)} .
$$

This is possible because of Theorem 2.18 (it suffices to choose $1+c_{1} / 2 \geq C_{1}^{2}$ ). Let $\delta=\delta\left(\Psi, \gamma_{1}, \gamma_{2}\right):=$ $1-\frac{1}{2} C_{1}^{-1} \in(1 / 2,1)$, where $C_{1}$ is the constant from Theorem 2.18. We further define $\epsilon=$ $\epsilon\left(\Psi, \gamma_{1}, \gamma_{2}\right) \in(0,1 / 4)$ by

$$
\epsilon:=\frac{1-\delta}{20 c_{1}},
$$

and choose $k_{0}=k_{0}\left(\Psi, \gamma_{1}, \gamma_{2}, \kappa\right) \in \mathbb{N}$ large enough so that $\left(1-\xi^{k_{0}}\right)^{-1} \leq 2$ and $C_{4} \xi^{k_{0}} \leq \varepsilon\left(4 C_{1}\right)^{-1}$ where $C_{4} \geq 1$ and $\xi \in(0,1)$ are the constants from Lemma 3.3.

For $k=0,1, \ldots$, define

$$
r_{k}=(\kappa / 2)^{k_{0} k} r, \quad B_{k}=B\left(Q, r_{k}\right), \quad D_{k}=D \cap B_{k}, \quad \Pi_{k}=D_{k} \backslash D_{k+1}, \quad \Pi_{-1}=B_{0}^{c} .
$$

For $l=-1,0,1, \ldots, k-1$, let

$$
\begin{aligned}
u_{k}^{l}(x):=\mathbb{E}_{x}\left[u\left(X_{\tau_{D_{k}}}\right) ; X_{\tau_{D_{k}}} \in \Pi_{l}\right], & & x \in \mathbb{R}^{d}, \\
v_{k}^{l}(x):=\mathbb{E}_{x}\left[v\left(X_{\tau_{D_{k}}}\right) ; X_{\tau_{D_{k}}} \in \Pi_{l}\right], & & x \in \mathbb{R}^{d} .
\end{aligned}
$$

Apply Lemma 3.3 with $\tilde{r}=r_{l+1}$ instead of $r$. Then $r_{k}=(\kappa / 2)^{k_{0} k} r=(\kappa / 2)^{k_{0}(k-l-1)} \tilde{r}$, hence for $k=0,1,2, \ldots$ and $x \in D_{k}$

$$
u_{k}^{l}(x) \leq C_{4}\left(\xi^{k_{0}}\right)^{k-l-1} u(x), \quad l=-1,0,1, \ldots, k-2 .
$$

Then since $\sum_{l=-1}^{k-2}\left(\xi^{k_{0}}\right)^{k-1-l}=\xi^{k_{0}} \sum_{n=0}^{k-1}\left(\xi^{k_{0}}\right)^{n} \leq \xi^{k_{0}}\left(1-\xi^{k_{0}}\right)^{-1} \leq 2 \xi^{k_{0}}$, we have that for $k=$ $1,2, \ldots$ and $x \in D_{k}$,

$$
\sum_{l=-1}^{k-2} u_{k}^{l}(x) \leq 2 C_{4} \xi^{k_{0}} u(x) .
$$

Since $2 C_{4} \xi^{k_{0}} \leq \varepsilon / 2<1 / 2$, for $k=1,2, \ldots, l=-1,0,1, \ldots, k-2$, we have $\sum_{l=-1}^{k-2} u_{k}^{l}(x) \leq u_{k}^{k-1}(x)$ for all $x \in D_{k}$ and, by (3.10)

$$
u_{k}^{l}(x) \leq \epsilon^{k-1-l} u_{k}^{k-1}(x), \quad x \in D_{k} .
$$

By symmetry we also have that for $k=1,2, \ldots, l=-1,0,1, \ldots, k-2$,

$$
v_{k}^{l}(x) \leq \epsilon^{k-1-l} v_{k}^{k-1}(x), \quad x \in D_{k} .
$$

Define $\zeta=\zeta\left(\Psi, \gamma_{1}, \gamma_{2}\right) \in(1 / 2,1)$ by

$$
\zeta=\sqrt{\frac{1+\delta}{2}}
$$


We claim that for all $l=0,1, \ldots$

$$
\sup _{x \in D_{l}} \frac{u(x)}{v(x)} \leq\left(1+c_{1} \zeta^{l}\right) \inf _{x \in D_{l}} \frac{u(x)}{v(x)} .
$$

By Theorem 2.18 with $y=A_{r}(Q)$ we have that

$$
C_{1}^{-1} \leq \frac{u(x)}{v(x)} \leq C_{1}, \quad x \in D_{0}=D \cap B(Q, r) .
$$

We show that (3.13) and (3.14) imply the statement of the lemma. Indeed,

$$
\sup _{x \in D_{l}} \frac{u(x)}{v(x)}-\inf _{x \in D_{l}} \frac{u(x)}{v(x)} \leq c_{1} \zeta^{l} \inf _{x \in D_{l}} \frac{u(x)}{v(x)} \leq C_{1} c_{1} \zeta^{l}, \quad l=0,1, \ldots
$$

Since the right-hand side goes to zero as $l \rightarrow \infty$, the same is valid for the left-hand side proving that the limit $g=\lim _{D \ni x \rightarrow Q} \frac{u(x)}{v(x)}$ exists. Further, for $x \in D \cap B(Q, r)$ there is a unique $l \geq 1$ such that $x \in \Pi_{l-1} \subset D_{l-1}$. Thus $x \notin D_{l}$ implying $|x-Q| \geq r_{l}=(\kappa / 2)^{k_{0} l} r$, that is

$$
l \geq \frac{\log \frac{r}{|x-Q|}}{k_{0} \log \frac{2}{\kappa}}
$$

Let $\beta:=-\frac{\log \zeta}{k_{0} \log \frac{2}{\kappa}}$. Then $\beta=\beta\left(\Psi, \gamma_{1}, \gamma_{2}, \kappa\right)$ and

$$
\left|\frac{u(x)}{v(x)}-g\right| \leq C_{1} c_{1} \zeta^{l} \leq C_{1} c_{1} \zeta^{\frac{\log \frac{r}{|x-Q|}}{k_{0} \log \frac{2}{\kappa}}}=C_{1} c_{1}\left(\frac{r}{|x-Q|}\right)^{\frac{\log \zeta}{k_{0} \log \frac{2}{\kappa}}}=C_{1} c_{1}\left(\frac{|x-Q|}{r}\right)^{\beta} .
$$

We now prove (3.13) by induction. By (3.6) we wee that (3.13) holds for $l=0$. Again by (3.6) and the fact that $\zeta>1 / 2$ we have

$$
\sup _{x \in D_{1}} \frac{u(x)}{v(x)} \leq \sup _{x \in D_{0}} \frac{u(x)}{v(x)} \leq\left(1+\frac{c_{1}}{2}\right) \inf _{x \in D_{0}} \frac{u(x)}{v(x)} \leq\left(1+c_{1} \zeta\right) \inf _{x \in D_{0}} \frac{u(x)}{v(x)} \leq\left(1+c_{1} \zeta\right) \inf _{x \in D_{1}} \frac{u(x)}{v(x)}
$$

hence (3.13) holds also for $l=1$. Let $k=0,1, \ldots$ and assume that (3.13) holds for $l=0,1,2, \ldots, k$. By the definitions (3.7)-(3.8), and the regular harmonicity of $u$ and $v$, we have

$$
\begin{array}{ll}
u(x)=\sum_{l=-1}^{k} u_{k+1}^{l}(x), & x \in D_{k+1}, \\
v(x)=\sum_{l=-1}^{k} v_{k+1}^{l}(x), & x \in D_{k+1} .
\end{array}
$$

For any function $f$ on a set $A$ we define

$$
\mathrm{Osc}_{A} f=\sup _{x \in A} f(x)-\inf _{x \in A} f(x) .
$$


Let $g(x):=u_{k+1}^{k}(x) / v_{k+1}^{k}(x), x \in D_{k}$. We claim that

$$
\operatorname{Osc}_{D_{k+2}} g \leq \delta \operatorname{Osc}_{D_{k}} g
$$

Recall that $\delta=1-\frac{1}{2} C_{1}^{-1} \in(1 / 2,1)$. Let $m_{1}:=\inf _{x \in D_{k}} g(x)$ and $m_{2}:=\sup _{x \in D_{k}} g(x)$. By (3.7), (3.8) and Theorem 2.18, it holds that $0<m_{1} \leq m_{2}<\infty$. If $m_{1}=m_{2}$, then both $\mathrm{Osc}_{D_{k+2}} g$ and $\mathrm{Osc}_{D_{k}} g$ are zero so (3.17) holds trivially. Otherwise, let

$$
\widetilde{g}(x):=\frac{g(x)-m_{1}}{m_{2}-m_{1}}=\frac{u_{k+1}^{k}(x)-m_{1} v_{k+1}^{k}(x)}{\left(m_{2}-m_{1}\right) v_{k+1}^{k}(x)}, \quad x \in D_{k} .
$$

Note that $\widetilde{g}$ is the quotient of two non-negative functions regular harmonic in $D_{k+1}$ with respect to $X$. Clearly $\operatorname{Osc}_{D_{k}} \widetilde{g}=1$. Furthermore,

$$
\operatorname{Osc}_{D_{k+2}} g=\operatorname{Osc}_{D_{k+2}} \widetilde{g} \cdot \operatorname{Osc}_{D_{k}} g .
$$

This is clear from $g(x)=\left(m_{2}-m_{1}\right) \widetilde{g}(x)+m_{1}=\left(\operatorname{Osc}_{D_{k}} g\right) \widetilde{g}(x)+m_{1}$. If $\sup _{D_{k+2}} \widetilde{g}(x) \leq 1 / 2$, then $\operatorname{Osc}_{D_{k+2}} \widetilde{g} \leq 1 / 2$, and it follows from (3.18) that

$$
\operatorname{Osc}_{D_{k+2}} g \leq \frac{1}{2} \operatorname{Osc}_{D_{k}} g
$$

If, on the other hand, $\sup _{D_{k+2}} \widetilde{g}(x)>1 / 2$, we apply Theorem 2.18 to the functions $\widetilde{u}(x)=u_{k+1}^{k}(x)-$ $m_{1} v_{k+1}^{k}(x)$ and $\widetilde{v}(x)=v_{k+1}^{k}(x)$ to conclude that

$$
C_{1}^{-1} \frac{\widetilde{u}(y)}{\widetilde{v}(y)} \leq \frac{\widetilde{u}(x)}{\widetilde{v}(x)} \leq C_{1} \frac{\widetilde{u}(y)}{\widetilde{v}(y)}, \quad x, y \in D_{k+1} .
$$

This can be written as

$$
C_{1}^{-1} \widetilde{g}(y) \leq \widetilde{g}(x) \leq C_{1} \widetilde{g}(y), \quad x, y \in D_{k+1} .
$$

Hence, for all $x \in D_{k+2}$, we have

$$
\widetilde{g}(x) \geq C_{1}^{-1} \sup _{y \in D_{k+2}} \widetilde{g}(y) \geq \frac{1}{2} C_{1}^{-1}
$$

Therefore, $\inf _{y \in D_{k+2}} \widetilde{g}(y) \geq \frac{1}{2} C_{1}^{-1}$, and since $\widetilde{g} \leq 1$, we get that

$$
\operatorname{Osc}_{D_{k+2}} \widetilde{g} \leq 1-\frac{1}{2} C_{1}^{-1}=\delta .
$$

By (3.18) $-(3.20)$ we get (3.17).

We claim that

$$
\inf _{x \in D_{k+2}} \frac{u_{k+1}^{k}(x)}{v_{k+1}^{k}(x)} \geq \inf _{x \in D_{k+1}} \frac{u_{k+1}^{k}(x)}{v_{k+1}^{k}(x)} \geq \inf _{x \in D_{k}} \frac{u(x)}{v(x)} \geq \inf _{x \in D_{i}} \frac{u(x)}{v(x)}, \quad i=0, \ldots k .
$$


Indeed, let $\eta:=\inf _{x \in D_{k}} \frac{u(x)}{v(x)}$ so that $u(x) \geq \eta v(x)$ on $D_{k}$. Then for $x \in D_{k+1}$,

$$
u_{k+1}^{k}(x)=\mathbb{E}_{x}\left[u\left(X_{\tau_{D_{k+1}}}\right) ; X_{\tau_{D_{k+1}}} \in \Pi_{k}\right] \geq \eta \mathbb{E}_{x}\left[v\left(X_{\tau_{D_{k+1}}}\right) ; X_{\tau_{D_{k+1}}} \in \Pi_{k}\right]=\eta v_{k+1}^{k}(x),
$$

so the second inequality of (3.21) holds. The first and third equalities of (3.21) are trivial. Similarly we have

$$
\sup _{x \in D_{k+2}} \frac{u_{k+1}^{k}(x)}{v_{k+1}^{k}(x)} \leq \sup _{x \in D_{k+1}} \frac{u_{k+1}^{k}(x)}{v_{k+1}^{k}(x)} \leq \sup _{x \in D_{k}} \frac{u(x)}{v(x)} .
$$

Combining (3.21), (3.22) and (3.17) we get

$$
\frac{\sup _{x \in D_{k+2}} \frac{u_{k+1}^{k}(x)}{v_{k+1}^{k}(x)}}{\inf _{x \in D_{k+2}} \frac{u_{k+1}^{k}(x)}{v_{k+1}^{k}(x)}}-1 \leq \delta\left(\frac{\sup _{x \in D_{k}} \frac{u(x)}{v(x)}}{\inf _{x \in D_{k}} \frac{u(x)}{v(x)}}-1\right) .
$$

Using (3.23) and (3.13) with $l=k$ (the induction hypothesis) we obtain

$$
\sup _{x \in D_{k+2}} \frac{u_{k+1}^{k}(x)}{v_{k+1}^{k}(x)}=\left(1+c_{1} \delta \rho \zeta^{k}\right) \inf _{x \in D_{k+2}} \frac{u_{k+1}^{k}(x)}{v_{k+1}^{k}(x)}
$$

with a suitably chosen $\rho \in[0,1]$ (independent of $x \in D_{k+2}$ ). Indeed,

$$
M:=\frac{\sup _{x \in D_{k+2}} \frac{u_{k+1}^{k}(x)}{v_{k+1}^{k}(x)}}{\inf _{x \in D_{k+2}} \frac{u_{k+1}^{k}(x)}{v_{k+1}^{k}(x)}} \leq 1+\delta\left(\frac{\sup _{x \in D_{k}} \frac{u(x)}{v(x)}}{\inf _{x \in D_{k}} \frac{u(x)}{v(x)}}-1\right) \leq 1+c_{1} \delta \zeta^{k}
$$

Thus, $1 \leq M \leq 1+c_{1} \delta \zeta^{k}$ which implies that there exists $\rho=\rho\left(c_{1}, \zeta, k\right) \in[0,1]$ such that $M=$ $1+c_{1} \delta \rho \zeta^{k}$. Next, by symmetry and (3.24) we have

$$
\sup _{x \in D_{k+2}} \frac{v_{k+1}^{k}(x)}{u_{k+1}^{k}(x)}=\left(1+c_{1} \delta \rho \zeta^{k}\right) \inf _{x \in D_{k+2}} \frac{v_{k+1}^{k}(x)}{u_{k+1}^{k}(x)} .
$$

Combining (3.21) and (3.13) we get

$$
\sup _{x \in D_{l}} \frac{u(x)}{v(x)} \leq\left(1+c_{1} \zeta^{l}\right) \inf _{x \in D_{k+2}} \frac{u_{k+1}^{k}(x)}{v_{k+1}^{k}(x)}, \quad l=0,1, \ldots, k .
$$

Now we fix $x \in D_{k+2}$. Then by (3.15) and (3.16),

$$
\begin{aligned}
\frac{u(x)}{v(x)} & =\frac{\sum_{l=0}^{k} u_{k+1}^{l}(x)+u_{k+1}^{-1}(x)}{\sum_{l=0}^{k} v_{k+1}^{l}(x)+v_{k+1}^{-1}(x)} \\
& \leq \frac{\sum_{l=0}^{k} u_{k+1}^{l}(x)+\epsilon^{k+1} u_{k+1}^{k}(x)}{\sum_{l=0}^{k} v_{k+1}^{l}(x)} \\
& \leq\left(1+\epsilon^{k+1}\right) \frac{\sum_{l=0}^{k} u_{k+1}^{l}(x)}{\sum_{l=0}^{k} v_{k+1}^{l}(x)},
\end{aligned}
$$


where in the first inequality we used (3.11) with $l=-1$. Now we apply [2, Lemma 15] with

$$
\begin{aligned}
& u_{0}=u_{k+1}^{k}(x), \quad v_{0}=v_{k+1}^{k}(x), \quad a=\left(1+c_{1} \delta \rho \zeta^{k}\right) \inf _{y \in D_{k+2}} \frac{u_{k+1}^{k}(y)}{v_{k+1}^{k}(y)} \\
& u_{i}=u_{k+1}^{k-i}(x), \quad v_{i}=v_{k+1}^{k-i}(x), \quad b_{i}=\left(1+c_{1} \zeta^{k-i}\right) \inf _{y \in D_{k+2}} \frac{u_{k+1}^{k}(y)}{v_{k+1}^{k}(y)}, \quad \epsilon_{i}=\epsilon^{i}, \quad i=1, \ldots, k .
\end{aligned}
$$

We need to check the conditions of [2, Lemma 15]. [2, (5.16)], $a \leq b_{i}, i=1, \ldots, k$, is immediate. [2, (5.18)], $u_{0} \leq a v_{0}$, is true by (3.24). [2, (5.17)] consists of two parts: $u_{i} \leq b_{i} v_{i}$ and $v_{i} \leq \epsilon_{i} v_{0}$ for $i=1, \ldots, k$. The first part follows from

$$
\sup _{y \in D_{k+1}} \frac{u_{k+1}^{k-i}(y)}{v_{k+1}^{k-i}(y)} \leq \sup _{y \in D_{k-i}} \frac{u(y)}{v(y)} \leq\left(1+c_{1} \zeta^{k-i}\right) \inf _{y \in D_{k+2}} \frac{u_{k+1}^{k-i}(y)}{v_{k+1}^{k-i}(y)},
$$

where the first inequality is (3.22) and the second inequality is (3.26). The second part of [2, (5.17)] is precisely (3.12). Now we apply [2, Lemma 15] to conclude that

$$
\begin{aligned}
& \sum_{i=0}^{k} u_{k+1}^{k-i}(x) \\
& \quad \leq\left[\left(1+c_{1} \delta \rho \zeta^{k}\right) \inf _{y \in D_{k+2}} \frac{u_{k+1}^{k}(y)}{v_{k+1}^{k}(y)}+\sum_{i=1}^{k} c_{1}\left(\zeta^{k-i}-\delta \rho \zeta^{k}\right) \inf _{y \in D_{k+2}} \frac{u_{k+1}^{k}(y)}{v_{k+1}^{k}(y)} \cdot \epsilon^{i}\right] \cdot \sum_{i=0}^{k} v_{k+1}^{k-i}(x) \\
& \quad \leq\left[1+c_{1} \delta \rho \zeta^{k}+c_{1} \sum_{i=1}^{k} \zeta^{k-i} \epsilon^{i}\right] \inf _{y \in D_{k+2}} \frac{u_{k+1}^{k}(y)}{v_{k+1}^{k}(y)} \cdot \sum_{i=0}^{k} v_{k+1}^{k-i}(x) .
\end{aligned}
$$

Hence, letting $\tau:=\left(1+\epsilon^{k+1}\right)\left(1+c_{1} \delta \rho \zeta^{k}+c_{1} \sum_{i=1}^{k} \zeta^{k-i} \epsilon^{i}\right)$ and applying (3.27) we get

$$
\frac{u(x)}{v(x)} \leq\left(1+\epsilon^{k+1}\right) \frac{\sum_{i=0}^{k} u_{k+1}^{k-i}(x)}{\sum_{i=0}^{k} v_{k+1}^{k-i}(x)} \leq \tau \cdot \inf _{y \in D_{k+2}} \frac{u_{k+1}^{k}(y)}{v_{k+1}^{k}(y)} .
$$

By symmetry,

$$
\frac{v(x)}{u(x)} \leq \tau \cdot \inf _{y \in D_{k+2}} \frac{v_{k+1}^{k}(y)}{u_{k+1}^{k}(y)} .
$$

Now, (3.28), (3.24) and (3.29) imply that

$$
\begin{aligned}
\sup _{y \in D_{k+2}} \frac{u(y)}{v(y)} & \leq \tau \cdot \inf _{y \in D_{k+2}} \frac{u_{k+1}^{k}(y)}{v_{k+1}^{k}(y)} \\
& =\frac{\tau}{1+c_{1} \delta \rho \zeta^{k}} \sup _{y \in D_{k+2}} \frac{u_{k+1}^{k}(y)}{v_{k+1}^{k}(y)} \\
& \leq \frac{\tau^{2}}{1+c_{1} \delta \rho \zeta^{k}} \inf _{y \in D_{k+2}} \frac{u(y)}{v(y)} .
\end{aligned}
$$


The proof of the induction step will be finished by showing that

$$
\frac{\tau^{2}}{1+c_{1} \delta \rho \zeta^{k}} \leq 1+c_{1} \zeta^{k+2}
$$

First note that

$$
\tau=\left(1+\epsilon^{k+1}\right)\left(1+c_{1}\left(\delta \rho+\frac{\epsilon}{\zeta-\epsilon}\left(1-\left(\frac{\epsilon}{\zeta}\right)^{k}\right)\right) \zeta^{k}\right)
$$

Let

$$
\widetilde{\tau}:=\left(1+\epsilon^{k+1}\right)\left(1+c_{1}\left(\delta \rho+\frac{\epsilon}{\zeta-\epsilon}\right) \zeta^{k}\right)
$$

so that $\tau \leq \widetilde{\tau}$. Then

$$
\begin{aligned}
\tau^{2} \leq \widetilde{\tau}^{2} & =\left(1+\epsilon^{k+1}\right)^{2}\left(1+2 c_{1}\left(\delta \rho+\frac{\epsilon}{\zeta-\epsilon}\right) \zeta^{k}+c_{1}^{2}\left(\delta \rho+\frac{\epsilon}{\zeta-\epsilon}\right)^{2} \zeta^{2 k}\right) \\
& \leq\left(1+3 \epsilon^{k+1}\right) A
\end{aligned}
$$

where

$$
A:=1+c_{1}\left(2 \delta \rho+\frac{2 \epsilon}{\zeta-\epsilon}\right) \zeta^{k}+c_{1}^{2}\left(\delta \rho+\frac{\epsilon}{\zeta-\epsilon}\right)^{2} \zeta^{2 k}
$$

Further, let

$$
B:=\left(1+c_{1} \delta \rho \zeta^{k}\right)\left(1+c_{1} \zeta^{k+2}\right)=1+c_{1}\left(\delta \rho+\zeta^{2}\right) \zeta^{k}+c_{1}^{2}\left(\delta \rho \zeta^{2}\right) \zeta^{2 k} .
$$

We will show that $B-\left(1+3 \epsilon^{k+1}\right) A=B-A-3 \epsilon^{k+1} A \geq 0$. Since $B-\left(1+3 \epsilon^{k+1}\right) A \leq B-\tau^{2}$, this will prove what want. We now prove $B-\left(1+3 \epsilon^{k+1}\right) A=B-A-3 \epsilon^{k+1} A \geq 0$. Note that $\epsilon \in(0,1 / 200) \subset(0,1 / 4)$ and thus $\zeta-\epsilon>1 / 2-1 / 4=1 / 4$. We will need the following estimate:

$$
\frac{\epsilon}{\zeta-\epsilon} \leq 4 \epsilon<1 \text {. }
$$

Since $\delta \in(1 / 2,1), \rho \in[0,1]$ and $\zeta<1$ we have

$$
A \leq 1+c_{1}(2+2)+c_{1}^{2}(1+1)^{2}=1+4 c_{1}+4 c_{1}^{2}=\left(1+2 c_{1}\right)^{2} \leq\left(3 c_{1}\right)^{2}=9 c_{1}^{2} .
$$

Note that it follows from (3.31) that

$$
\zeta^{2}-\delta-8 \epsilon=\frac{1+\delta}{2}-\delta-\frac{2(1-\delta)}{5 c_{1}}=(1-\delta)\left(\frac{1}{2}-\frac{2}{5 c_{1}}\right) \geq \frac{2}{5}(1-\delta),
$$

where we used that $1 / 2-2 /\left(5 c_{1}\right) \geq 1 / 2-2 / 50=23 / 50 \geq 2 / 5$ which follows immediately from $c_{1} \geq 10$. Thus,

$$
\begin{aligned}
B-A & =c_{1}\left(\delta \rho+\zeta^{2}-2 \delta \rho-\frac{2 \epsilon}{\zeta-\epsilon}\right) \zeta^{k}+c_{1}^{2}\left(\delta \rho \zeta^{2}-\delta^{2} \rho^{2}-2 \delta \rho \frac{\epsilon}{\zeta-\epsilon}-\frac{\epsilon^{2}}{(\zeta-\epsilon)^{2}}\right) \zeta^{2 k} \\
& =c_{1}\left(\zeta^{2}-\delta \rho-\frac{2 \epsilon}{\zeta-\epsilon}\right) \zeta^{k}+c_{1}^{2}\left[\delta \rho\left(\zeta^{2}-\delta \rho-\frac{2 \epsilon}{\zeta-\epsilon}\right)-\frac{\epsilon^{2}}{(\zeta-\epsilon)^{2}}\right] \zeta^{2 k}
\end{aligned}
$$




$$
\begin{aligned}
& >c_{1}\left(\zeta^{2}-\delta \rho-8 \epsilon\right) \zeta^{k}-c_{1}^{2} \frac{\epsilon^{2}}{(\zeta-\epsilon)^{2}} \zeta^{2 k} \\
& \geq c_{1}\left(\zeta^{2}-\delta-8 \epsilon\right) \zeta^{k}-c_{1}^{2}(4 \epsilon)^{2} \zeta^{2 k} \\
& \geq \frac{2}{5} c_{1}(1-\delta)\left(\frac{1+\delta}{2}\right)^{k / 2}-c_{1}^{2}\left(\frac{1-\delta}{5 c_{1}}\right)^{2}\left(\frac{1+\delta}{2}\right)^{k} \\
& =\frac{2}{5} c_{1}(1-\delta)\left(\frac{1+\delta}{2}\right)^{k / 2}-\frac{(1-\delta)^{2}}{25}\left(\frac{1+\delta}{2}\right)^{k} \\
& =(1-\delta)\left(\frac{1+\delta}{2}\right)^{k / 2}\left(\frac{2}{5} c_{1}-\frac{1-\delta}{25}\left(\frac{1+\delta}{2}\right)^{k / 2}\right) \\
& \geq(1-\delta)\left(\frac{1+\delta}{2}\right)^{k / 2}\left(4-\frac{1}{25}\left(\frac{1+\delta}{2}\right)^{k / 2}\right)
\end{aligned}
$$

where in the third line above we used (3.31) and neglected the first term in the square brackets in the line above, in the fourth line we used that $\rho \leq 1$ and again (3.31), and in the last line we used that $c_{1} \geq 10$.

By combining (3.32) and (3.33) we get

$$
\begin{aligned}
B-A-3 \epsilon^{k+1} A & \geq B-A-3 \epsilon^{k+1} \cdot 9 c_{1}^{2} \\
& \geq(1-\delta)\left(\frac{1+\delta}{2}\right)^{k / 2}\left(4-\frac{1}{25}\left(\frac{1+\delta}{2}\right)^{k / 2}\right)-27\left(\frac{1-\delta}{20 c_{1}}\right)^{k-1}\left(\frac{1-\delta}{20 c_{1}}\right)^{2} c_{1}^{2} \\
& =(1-\delta)\left(\frac{1+\delta}{2}\right)^{k / 2}\left(4-\frac{1}{25}\left(\frac{1+\delta}{2}\right)^{k / 2}\right)-\frac{27}{20^{2}}\left(\frac{1-\delta}{20 c_{1}}\right)^{k-1}(1-\delta)^{2} \\
& \geq(1-\delta)\left(\frac{1+\delta}{2}\right)^{k / 2}\left(4-\frac{1}{25}\left(\frac{1+\delta}{2}\right)^{k / 2}\right)-27\left(\frac{1-\delta}{20}\right)^{k+1} \\
& \geq 3(1-\delta)\left(\frac{1+\delta}{2}\right)^{k / 2}-27\left(\frac{1-\delta}{20}\right)^{k+1} \\
& \geq 3 \frac{1}{2}\left(\frac{1}{2}\right)^{k / 2}-27\left(\frac{1}{20}\right)^{k+1} \\
& =3\left(\frac{1}{2}\right)^{k / 2+1}-27\left(\frac{1}{20}\right)^{k+1}>0
\end{aligned}
$$

for all $k \geq 1$. In the penultimate line we used that $1-\delta \geq 1 / 2,(1+\delta) / 2 \geq 1 / 2$ and $1-\delta<1$.

The proof is now complete.

An alternative approach to the oscillation reduction in case of rotationally stable processes is given in [6, Lemma 8]. We note that for non-local operators the oscillation reduction technique seems much harder than for the Laplacian, because the subtraction used in this process may destroy global nonnegativity. In this regard, we note that there is a gap of this nature in the proof of [11, Lemma 3.3]. 
In the remainder of this section, we assume that $Q \in \partial D$. Since $D$ is Greenian, the Green function $G_{D}(x, y), x, y \in D$, is well defined. Fix $x_{0} \in D$ and set

$$
M_{D}(x, y)=\frac{G_{D}(x, y)}{G_{D}\left(x_{0}, y\right)}, \quad x, y \in D, y \neq x_{0}
$$

Let $r<\frac{1}{2} \min \left\{\operatorname{dist}(x, Q)\right.$, dist $\left.\left(x_{0}, Q\right)\right\}$. Since $G_{D}(x, \cdot)$ and $G_{D}\left(x_{0}, \cdot\right)$ are regular harmonic in $D \cap$ $B(Q, 2 r)$ and vanish in $D^{c} \cap B(Q, 2 r)$, using Lemma 3.4, one immediately gets the following.

Corollary 3.5 The limit $M_{D}(x, Q):=\lim _{D \ni y \rightarrow Q} M_{D}(x, y)$ exists. Furthermore, there exist positive constants $c$ and $\beta$ depending on $\left(\Psi, \gamma_{1}, \gamma_{2}, \kappa\right)$ such that for any $r \in\left(0, \frac{1}{2}\left(R \wedge \operatorname{dist}\left(x_{0}, Q\right)\right)\right.$, any $y \in D \cap B(Q, r)$ and any $x \in D \backslash B(Q, 2 r)$,

$$
\left|M_{D}(x, y)-M_{D}(x, Q)\right| \leq c M_{D}\left(x, A_{r}(Q)\right)\left(\frac{|y-Q|}{r}\right)^{\beta} .
$$

Proof. Put

$$
u(y):=\frac{G_{D}(x, y)}{G_{D}\left(x, A_{r}(Q)\right)}, \quad v(y):=\frac{G_{D}\left(x_{0}, y\right)}{G_{D}\left(x_{0}, A_{r}(Q)\right)} .
$$

Since $u$ and $v$ satisfy the assumptions of Lemma 3.4, there exists

$$
g:=\lim _{D \ni y \rightarrow Q} \frac{u(y)}{v(y)}=\lim _{D \ni y \rightarrow Q} \frac{M_{D}(x, y)}{M_{D}\left(x, A_{r}(Q)\right)} .
$$

This implies the existence of $M_{D}(x, Q):=\lim _{D \ni y \rightarrow Q} M_{D}(x, y)$. Furthermore, by (3.5) we have that

$$
\left|\frac{u(y)}{v(y)}-g\right| \leq c\left(\frac{|y-Q|}{r}\right)^{\beta}, \quad \text { for all } y \in D \cap B(Q, r) \text {. }
$$

Equivalently,

$$
\left|\frac{M_{D}(x, y)}{M_{D}\left(x, A_{r}(Q)\right)}-\frac{M_{D}(x, Q)}{M_{D}\left(x, A_{r}(Q)\right)}\right| \leq c\left(\frac{|y-Q|}{r}\right)^{\beta}, \quad \text { for all } y \in D \cap B(Q, r),
$$

which is (3.34).

Recall that $X^{D}$ is the process $X$ killed upon exiting $D$. As the process $X^{D}$ satisfies Hypothesis (B) in [25], $D$ has a Martin boundary $\partial_{M} D$ with respect to $X^{D}$ satisfying the following properties:

(M1) $D \cup \partial_{M} D$ is a compact metric space (with the metric denoted by $d$ );

(M2) $D$ is open and dense in $D \cup \partial_{M} D$, and its relative topology coincides with its original topology;

(M3) $M_{D}(x, \cdot)$ can be uniquely extended to $\partial_{M} D$ in such a way that

(a) $M_{D}(x, y)$ converges to $M_{D}(x, w)$ as $y \rightarrow w \in \partial_{M} D$ in the Martin topology; 
(b) for each $w \in D \cup \partial_{M} D$ the function $x \rightarrow M_{D}(x, w)$ is excessive with respect to $X^{D}$;

(c) the function $(x, w) \rightarrow M_{D}(x, w)$ is jointly continuous on $D \times\left(D \cup \partial_{M} D\right)$ in the Martin topology and

(d) $M_{D}\left(\cdot, w_{1}\right) \neq M_{D}\left(\cdot, w_{2}\right)$ if $w_{1} \neq w_{2}$ and $w_{1}, w_{2} \in \partial_{M} D$.

We will say that a point $w \in \partial_{M} D$ is a finite Martin boundary point if there exists a bounded sequence $\left(y_{n}\right)_{n \geq 1} \subset D$ converging to $w$ in the Martin topology. The finite part of the Martin boundary will be denoted by $\partial_{M}^{f} D$. Recall that a point $w$ on the Martin boundary $\partial_{M} D$ of $D$ is said to be associated with $Q \in \partial D$ if there is a sequence $\left(y_{n}\right)_{n \geq 1} \subset D$ converging to $w$ in the Martin topology and to $Q$ in the Euclidean topology. The set of Martin boundary points associated with $Q$ is denoted by $\partial_{M}^{Q} D$.

Proposition 3.6 $\partial_{M}^{Q} D$ consists of exactly one point.

Proof. We first note that $\partial_{M}^{Q} D$ is not empty. Indeed, let $\left(y_{n}\right)_{n \geq 1} \subset D$ converge to $Q$ in the Euclidean topology. Since $D \cup \partial_{M} D$ is a compact metric space with the Martin metric, there exist a subsequence $\left(y_{n_{k}}\right)_{k \geq 1}$ and $w \in D \cup \partial_{M} D$ such that $\lim _{k \rightarrow \infty} d\left(y_{n_{k}}, w\right)=0$. Clearly, $w \notin D$ (since relative topologies on $D$ are equivalent). Thus we have found a sequence $\left(y_{n_{k}}\right)_{k \geq 1} \subset D$ which converges to $w \in \partial_{M} D$ in the Martin topology and to $Q$ in the Euclidean topology.

Let $w \in \partial_{M}^{Q} D$ and let $M_{D}(\cdot, w)$ be the corresponding Martin kernel. If $\left(y_{n}\right)_{n \geq 1} \subset D$ is a sequence converging to $w$ in the Martin topology and to $Q$ in the Euclidean topology, then, by (M3)(a), $M_{D}\left(x, y_{n}\right)$ converge to $M_{D}(x, w)$. On the other hand, $\left|y_{n}-Q\right| \rightarrow 0$, thus by Corollary 3.5 ,

$$
\lim _{n \rightarrow \infty} M_{D}\left(x, y_{n}\right)=M_{D}(x, Q) .
$$

Hence, for each $w \in \partial_{M}^{Q} D$ it holds that $M_{D}(\cdot, w)=M_{D}(\cdot, Q)$. Since, by (M3)(d), for two different Martin boundary points $w^{(1)}$ and $w^{(2)}$ it always holds that $M_{D}\left(\cdot, w^{(1)}\right) \neq M_{D}\left(\cdot, w^{(2)}\right)$, we conclude that $\partial_{M}^{Q} D$ consists of exactly one point.

Because of the proposition above, we will also use $Q$ to denote the point on the Martin boundary $\partial_{M}^{Q} D$ associated with $Q$. Note that it follows from the proof of Proposition 3.6 that if $\left(y_{n}\right)_{n \geq 1}$ converges to $Q$ in the Euclidean topology, then it also converges to $Q$ in the Martin topology.

For $\epsilon>0$ let

$$
K_{\epsilon}:=\left\{w \in \partial_{M} D: d(w, Q) \geq \epsilon\right\}
$$

be a closed subset of $\partial_{M} D$. By the definition of Martin boundary, for each $w \in K_{\epsilon}$ there exists a sequence $\left(y_{n}^{w}\right)_{n \geq 1} \subset D$ such that $\lim _{n \rightarrow \infty} d\left(y_{n}^{w}, w\right)=0$. Without loss of generality we may assume that $d\left(y_{n}^{w}, w\right)<\frac{\epsilon}{2}$ for all $n \geq 1$.

Lemma 3.7 There exists $c=c(\epsilon)>0$ such that $\left|y_{n}^{w}-Q\right| \geq c$ for all $w \in K_{\epsilon}$ and all $n \geq 1$. 
Proof. Suppose the lemma is not true. Then $\left\{y_{n}^{w}: w \in K_{\epsilon}, n \in \mathbb{N}\right\}$ contains a sequence $\left(y_{n_{k}}^{w_{k}}\right)_{k \geq 1}$ such that $\lim _{k \rightarrow \infty}\left|y_{n_{k}}^{w_{k}}-Q\right|=0$. Then also $\lim _{k \rightarrow \infty} d\left(y_{n_{k}}^{w_{k}}, Q\right)=0$. On the other hand,

$$
d\left(y_{n_{k}}^{w_{k}}, Q\right) \geq d\left(w_{k}, Q\right)-d\left(y_{n_{k}}^{w_{k}}, w_{k}\right) \geq \epsilon-\frac{\epsilon}{2}=\frac{\epsilon}{2} .
$$

This contradiction proves the claim.

We continue by showing that $M_{D}(\cdot, Q)$ is harmonic in $D$ with respect to $X$.

Lemma 3.8 For every bounded open $U \subset \bar{U} \subset D$ and every $x \in D, M_{D}\left(X_{\tau_{U}}, Q\right)$ is $\mathbb{P}_{x}$-integrable.

Proof. Let $\left(y_{m}\right)_{m \geq 1}$ be a sequence in $D \backslash \bar{U}$ such that $\left|y_{m}-Q\right| \rightarrow 0$. Then $M_{D}\left(\cdot, y_{m}\right)$ is regular harmonic in $U$. Hence, by Fatou's lemma and Corollary 3.5 .

$$
\begin{aligned}
& \mathbb{E}_{x}\left[M_{D}\left(X_{\tau_{U}}, Q\right)\right]=\mathbb{E}_{x}\left[\lim _{m \rightarrow \infty} M_{D}\left(X_{\tau_{U}}, y_{m}\right)\right] \leq \liminf _{m \rightarrow \infty} \mathbb{E}_{x}\left[M_{D}\left(X_{\tau_{U}}, y_{m}\right)\right] \\
& =\liminf _{m \rightarrow \infty} M_{D}\left(x, y_{m}\right)=M_{D}(x, Q)<\infty
\end{aligned}
$$

Using the results above, we can get the following result.

Lemma 3.9 Suppose that $D$ is either (i) a bounded open set or (ii) an unbounded open set and $X$ is transient. For any $x \in D$ and $\rho \in\left(0, R \wedge\left(\delta_{D}(x) / 2\right)\right]$,

$$
M_{D}(x, Q)=\mathbb{E}_{x}\left[M_{D}\left(X_{\tau_{B(x, \rho)}}, Q\right)\right] .
$$

Proof. Fix $x \in D$ and a positive $r<R \wedge \frac{\delta_{D}(x)}{2}$. Let

$$
\eta_{m}:=\left(\frac{\kappa}{2}\right)^{m} r \quad \text { and } \quad z_{m}=A_{\eta_{m}}(Q), \quad m=0,1, \ldots
$$

Note that

$$
B\left(z_{m}, \eta_{m+1}\right) \subset D \cap B\left(Q, \frac{\eta_{m}}{2}\right) \subset D \cap B\left(Q, \eta_{m}\right) \subset D \cap B(Q, r) \subset D \backslash B(x, r)
$$

for all $m \geq 0$. Thus by the harmonicity of $M_{D}\left(\cdot, z_{m}\right)$, we have

$$
M_{D}\left(x, z_{m}\right)=\mathbb{E}_{x}\left[M_{D}\left(X_{\tau_{B(x, r)}}, z_{m}\right)\right] .
$$

On the other hand, by Theorem 2.18, there exist $m_{0}=m_{0}(\kappa) \geq 2$ and $c_{1}=c_{1}\left(\Psi, \gamma_{1}, \gamma_{2}, \kappa\right)>0$ such that for every $w \in D \backslash B\left(Q, \eta_{m}\right)$ and $y \in D \cap B\left(Q, \eta_{m+1}\right)$,

$$
M_{D}\left(w, z_{m}\right)=\frac{G_{D}\left(w, z_{m}\right)}{G_{D}\left(x_{0}, z_{m}\right)} \leq c_{1} \frac{G_{D}(w, y)}{G_{D}\left(x_{0}, y\right)}=c_{1} M_{D}(w, y), \quad m \geq m_{0} .
$$


Letting $y \rightarrow Q$ we get

$$
M_{D}\left(w, z_{m}\right) \leq c_{1} M_{D}(w, Q), \quad m \geq m_{0}, w \in D \backslash B\left(Q, \eta_{m}\right) .
$$

To prove this lemma, it suffices to show that $\left\{M_{D}\left(X_{\tau_{B(x, r)}}, z_{m}\right): m \geq m_{0}\right\}$ is $\mathbb{P}_{x^{-}}$-uniformly integrable. Since $M_{D}\left(X_{\tau_{B(x, r)}}, Q\right)$ is integrable by Lemma [3.8, for any $\epsilon>0$, there is an $N_{0}>1$ such that

$$
\mathbb{E}_{x}\left[M_{D}\left(X_{\tau_{B(x, r)}}, Q\right) ; M_{D}\left(X_{\tau_{B(x, r)}}, Q\right)>N_{0} / c_{1}\right]<\frac{\epsilon}{2 c_{1}}
$$

Note that by (3.36) and (3.37),

$$
\begin{aligned}
& \mathbb{E}_{x}\left[M_{D}\left(X_{\tau_{B(x, r)}}, z_{m}\right) ; M_{D}\left(X_{\tau_{B(x, r)}}, z_{m}\right)>N_{0} \text { and } X_{\tau_{B(x, r)}} \in D \backslash B\left(Q, \eta_{m}\right)\right] \\
& \leq c_{1} \mathbb{E}_{x}\left[M_{D}\left(X_{\tau_{B(x, r)}}, Q\right) ; c_{1} M_{D}\left(X_{\tau_{B(x, r)}}, Q\right)>N_{0}\right]<c_{1} \frac{\epsilon}{2 c_{1}}=\frac{\epsilon}{2} .
\end{aligned}
$$

By (2.9), we have for $m \geq m_{0}$,

$$
\begin{aligned}
& \mathbb{E}_{x}\left[M_{D}\left(X_{\tau_{B(x, r)}}, z_{m}\right) ; X_{\tau_{B(x, r)}} \in D \cap B\left(Q, \eta_{m}\right)\right] \\
& =\int_{D \cap B\left(Q, \eta_{m}\right)} M_{D}\left(w, z_{m}\right) K_{B(x, r)}(x, w) d w \\
& \leq c_{2} \phi\left(r^{-2}\right)^{-1} \int_{D \cap B\left(Q, \eta_{m}\right)} M_{D}\left(w, z_{m}\right) j(|w-x|-r) d w
\end{aligned}
$$

for some $c_{2}=c_{2}\left(\Psi, \gamma_{1}, \gamma_{2}\right)>0$. Since $|w-x| \geq|x-Q|-|Q-w| \geq \delta_{D}(x)-\eta_{m} \geq \frac{7}{4} r$, using (2.4) and (2.2), we get that

$$
\begin{aligned}
& \mathbb{E}_{x}\left[M_{D}\left(X_{\tau_{B(x, r)}}, z_{m}\right) ; X_{\tau_{B(x, r)}} \in D \cap B\left(Q, \eta_{m}\right)\right] \\
& \leq c_{3} j(r) \phi\left(r^{-2}\right)^{-1} \int_{D \cap B\left(Q, \eta_{m}\right)} M_{D}\left(w, z_{m}\right) d w \\
& \leq c_{4} r^{-d} \int_{D \cap B\left(Q, \eta_{m}\right)} M_{D}\left(w, z_{m}\right) d w \\
& =c_{4} r^{-d} G_{D}\left(x_{0}, z_{m}\right)^{-1} \int_{D \cap B\left(Q, \eta_{m}\right)} G_{D}\left(w, z_{m}\right) d w
\end{aligned}
$$

for some $c_{3}=c_{3}\left(\Psi, \gamma_{1}, \gamma_{2}\right)>0$ and $c_{4}=c_{4}\left(\Psi, \gamma_{1}, \gamma_{2}\right)>0$. Note that, by Lemma 3.1,

$$
G_{D}\left(x_{0}, z_{m}\right)^{-1} \leq C_{3}\left(\phi\left(\eta_{m_{0}}^{-2}\right)\right)^{-1} \xi^{m-m_{0}} \phi\left(\eta_{m}^{-2}\right) G_{D}\left(x_{0}, z_{m_{0}}\right)^{-1}
$$

By (2.3), there exists $c_{5}=c_{5}\left(\Psi, \gamma_{1}, \gamma_{2}\right)>0$ such that for any $\eta<1$,

$$
\int_{0}^{\eta} \frac{1}{s \phi\left(s^{-2}\right)} d s \leq c_{5} \phi\left(\eta^{-2}\right)^{-1}
$$

Thus by Lemma 2.12 in case $D$ is bounded and by Theorem 2.19 in case of unbounded $D$,

$$
\int_{B\left(Q, \eta_{m}\right)} G_{D}\left(w, z_{m}\right) d w \leq c_{6} \int_{B\left(z_{m}, 2 \eta_{m}\right)} \frac{1}{\left|w-z_{m}\right|^{d} \phi\left(\left|w-z_{m}\right|^{-2}\right)} d w \leq c_{7} \phi\left(\left(2 \eta_{m}\right)^{-2}\right)^{-1}
$$


for some constants $c_{6}=c_{6}\left(\Psi, \gamma_{1}, \gamma_{2}\right)>0$ and $c_{7}=c_{7}\left(\Psi, \gamma_{1}, \gamma_{2}\right)>0$. It follows from (3.38) -(3.40) that

$$
\begin{aligned}
& \mathbb{E}_{x}\left[M_{D}\left(X_{\tau_{B(x, r)}}, z_{m}\right) ; X_{\tau_{B(x, r)}} \in D \cap B\left(Q, \eta_{m}\right)\right] \\
& \leq c_{8} r^{-d}\left(\phi\left(\eta_{m_{0}}^{-2}\right)\right)^{-1} G_{D}\left(x_{0}, z_{m_{0}}\right)^{-1} \frac{\phi\left(\eta_{m}^{-2}\right)}{\phi\left(\left(2 \eta_{m}\right)^{-2}\right)} \xi^{m-m_{0}} .
\end{aligned}
$$

Applying Lemma 2.1, we get

$$
\mathbb{E}_{x}\left[M_{D}\left(X_{\tau_{B(x, r)}}, z_{m}\right) ; X_{\tau_{B(x, r)}} \in D \cap B\left(Q, \eta_{m}\right)\right] \leq c_{9} r^{-d}\left(\phi\left(\eta_{m_{0}}^{-2}\right)\right)^{-1} G_{D}\left(x_{0}, z_{m_{0}}\right)^{-1} \xi^{m-m_{0}} .
$$

Thus there exists $N>0$ such that for all $m \geq N$,

$$
\mathbb{E}_{x}\left[M_{D}\left(X_{\tau_{B(x, r)}}, z_{m}\right) ; X_{\tau_{B(x, r)}} \in D \cap B\left(Q, \eta_{m}\right)\right] \leq \frac{\epsilon}{2} .
$$

Consequently, for all $m \geq N$,

$$
\mathbb{E}_{x}\left[M_{D}\left(X_{\tau_{B(x, r)}}, z_{m}\right) ; M_{D}\left(X_{\tau_{B(x, r)}}, z_{m}\right)>N\right] \leq \epsilon,
$$

which implies that $\left\{M_{D}\left(X_{\tau_{B(x, r)}}, z_{m}\right): m \geq m_{0}\right\}$ is $\mathbb{P}_{x^{-}}$uniformly integrable.

Using this, we can easily get the following

Theorem 3.10 The function $M_{D}(\cdot, Q)$ is harmonic in $D$ with respect to $X$.

Proof. The proof is basically the same as that of [19, Theorem 3.9]. We write the details here for completeness. Let $h(x):=M_{D}(x, Q)$. Consider a relatively compact open set $D_{1} \subset \overline{D_{1}} \subset D$, and put $r(x)=R \wedge\left(\frac{1}{3} \delta_{D}(x)\right)$ and $B(x)=B(x, r(x))$. Define a sequence $\left\{T_{m}: m \geq 1\right\}$ of stopping times as follows: $T_{1}:=\inf \left\{t>0: X_{t} \notin B\left(X_{0}\right)\right\}$, and for $m \geq 2$,

$$
T_{m}:= \begin{cases}T_{m-1}+\tau_{B\left(X_{T_{m-1}}\right)} \cdot \theta_{T_{m-1}} & \text { if } X_{T_{m-1}} \in D_{1} \\ \tau_{D_{1}} & \text { otherwise. }\end{cases}
$$

Note that $X_{\tau_{D_{1}}} \in \partial D_{1}$ on $\cap_{n=1}^{\infty}\left\{T_{n}<\tau_{D_{1}}\right\}$. Thus, since $\lim _{m \rightarrow \infty} T_{m}=\tau_{D_{1}} \mathbb{P}_{x^{-}}$.s.s. and $h$ is continuous in $D$, using the quasi-left continuity of $X^{D}$, we have $\lim _{m \rightarrow \infty} h\left(X_{T_{m}}^{D}\right)=h\left(X_{\tau_{D_{1}}}^{D}\right)$ on $\cap_{n=1}^{\infty}\left\{T_{n}<\tau_{D_{1}}\right\}$. Now by the dominated convergence theorem and Lemma 3.9.

$$
\begin{aligned}
h(x) & =\lim _{m \rightarrow \infty} \mathbb{E}_{x}\left[h\left(X_{T_{m}}^{D}\right) ; \cup_{n=1}^{\infty}\left\{T_{n}=\tau_{D_{1}}\right\}\right]+\lim _{m \rightarrow \infty} \mathbb{E}_{x}\left[h\left(X_{T_{m}}^{D}\right) ; \cap_{n=1}^{\infty}\left\{T_{n}<\tau_{D_{1}}\right\}\right] \\
& =\mathbb{E}_{x}\left[h\left(X_{\tau_{D_{1}}}^{D}\right) ; \cup_{n=1}^{\infty}\left\{T_{n}=\tau_{D_{1}}\right\}\right]+\mathbb{E}_{x}\left[h\left(X_{\tau_{D_{1}}}^{D}\right) ; \cap_{n=1}^{\infty}\left\{T_{n}<\tau_{D_{1}}\right\}\right] \\
& =\mathbb{E}_{x}\left[h\left(X_{\tau_{D_{1}}}^{D}\right)\right] .
\end{aligned}
$$

Part (b) of the following result is proved in [23, Lemma 4.18]. Part (a) is even simpler. 
Lemma 3.11 (a) Let $D$ be a bounded open set and suppose that $u$ is a bounded nonnegative harmonic function for $X^{D}$. If there exists a polar set $N \subset \partial D$ such that for any $z \in \partial D \backslash N$

$$
\lim _{D \ni x \rightarrow z} u(x)=0
$$

then $u$ is identically equal to zero.

(b) Let $D$ be an unbounded open set and suppose that $u$ is a bounded nonnegative harmonic function for $X^{D}$. If there exists a polar set $N \subset \partial D$ such that for any $z \in \partial D \backslash N$ (3.41) holds true and additionally

$$
\lim _{D \ni x \rightarrow \infty} u(x)=0
$$

then $u$ is identically equal to zero.

The next result completes the proof of Theorem 1.2, Recall that a point $z \in \partial D$ is said to regular boundary point of $D$ if $\mathbb{P}_{z}\left(\tau_{D}=0\right)=1$ and an irregular boundary point if $\mathbb{P}_{z}\left(\tau_{D}=0\right)=0$. The set of irregular boundary points is polar.

Theorem 3.12 Assume that either $D$ is bounded, or $D$ is unbounded and $X$ is transient. Then $Q$ is a minimal Martin boundary point, that is, $M_{D}(\cdot, Q)$ is a minimal harmonic function.

Proof. Let $h$ be a positive harmonic function for $X^{D}$ such that $h \leq M_{D}(\cdot, Q)$. By the Martin representation in [25], there is a finite measure on $\partial_{M} D$ such that

$$
h(x)=\int_{\partial_{M} D} M_{D}(x, w) \mu(d w)=\int_{\partial_{M} D \backslash\{Q\}} M_{D}(x, w) \mu(d w)+M_{D}(x, Q) \mu(\{Q\}) .
$$

In particular, $\mu\left(\partial_{M} D\right)=h\left(x_{0}\right) \leq M_{D}\left(x_{0}, Q\right)=1$ (because of the normalization at $x_{0}$ ). Hence, $\mu$ is a sub-probability measure.

For $\epsilon>0, K_{\epsilon}$ is the compact subset of $\partial_{M} D$ defined in (3.35). Define

$$
u(x):=\int_{K_{\epsilon}} M_{D}(x, w) \mu(d w) .
$$

Then $u$ is a positive harmonic function with respect to $X^{D}$ satisfying

$$
u(x) \leq h(x)-\mu(\{Q\}) M_{D}(x, Q) \leq(1-\mu(\{Q\})) M_{D}(x, Q) .
$$

Let $c=c(\epsilon)>0$ be the constant from Lemma 3.7. Hence, for $w \in K_{\epsilon}$ and $\left(y_{n}^{w}\right)_{n \geq 1}$ a sequence such that $\lim _{n \rightarrow \infty} d\left(y_{n}^{w}, w\right)=0$, it holds that $\left|y_{n}^{w}-Q\right| \geq c$. Fix $x_{1} \in D \cap B(Q, c / 2)$ and choose arbitrary $y_{0} \in D \backslash B(Q, c)$. For any $x \in D \cap B(Q, c / 2)$ and any $y \in D \backslash B(Q, c)$ we have that

$$
\frac{G_{D}(x, y)}{G_{D}\left(x_{0}, y\right)}=\frac{G_{D}(x, y)}{G_{D}\left(x_{1}, y\right)} \frac{G_{D}\left(x_{1}, y\right)}{G_{D}\left(x_{0}, y\right)} \leq c_{1} \frac{G_{D}\left(x, y_{0}\right)}{G_{D}\left(x_{1}, y_{0}\right)} \frac{G_{D}\left(x_{1}, y\right)}{G_{D}\left(x_{0}, y\right)} .
$$


Here the inequality follows from Theorem 2.18 applied to functions $G_{D}(\cdot, y)$ and $G_{D}\left(\cdot, y_{0}\right)$ which are regular harmonic in $D \cap B(Q, c)$ and vanish in $D \backslash B(Q, c)$. Now fix $w \in K_{\epsilon}$ and apply the above inequality to $y_{n}^{w}$ to get

$$
\begin{aligned}
M_{D}(x, w) & =\lim _{n \rightarrow \infty} \frac{G_{D}\left(x, y_{n}^{w}\right)}{G_{D}\left(x_{0}, y_{n}^{w}\right)} \leq c_{1} \frac{G_{D}\left(x, y_{0}\right)}{G_{D}\left(x_{1}, y_{0}\right)} \lim _{n \rightarrow \infty} \frac{G_{D}\left(x_{1}, y_{n}^{w}\right)}{G_{D}\left(x_{0}, y_{n}^{w}\right)} \\
& =c_{1} \frac{G_{D}\left(x, y_{0}\right)}{G_{D}\left(x_{1}, y_{0}\right)} M_{D}\left(x_{1}, w\right) \leq c_{1} \frac{G_{D}\left(x, y_{0}\right)}{G_{D}\left(x_{1}, y_{0}\right)} \sup _{w \in K_{\epsilon}} M_{D}\left(x_{1}, w\right) \\
& \leq c_{2} \frac{G_{D}\left(x, y_{0}\right)}{G_{D}\left(x_{1}, y_{0}\right)}=c_{3} G_{D}\left(x, y_{0}\right) .
\end{aligned}
$$

In the last line we used property (M3) (c) of the Martin kernel. Thus,

$$
M_{D}(x, w) \leq c_{3} G_{D}\left(x, y_{0}\right), \quad x \in D \cap B(Q, c / 2), w \in K_{\epsilon} .
$$

Choose $r<c / 4$. For any $x \in D \backslash B(Q, 2 r)$ and $y \in D \cap B(Q, r)$ with $r$ small enough, by Theorem 2.18 applied to $G_{D}(x, \cdot)$ and $G_{D}\left(x_{0}, \cdot\right)$, we have

$$
\frac{G_{D}(x, y)}{G_{D}\left(x_{0}, y\right)} \leq c_{4} \frac{G_{D}\left(x, A_{r}(Q)\right)}{G_{D}\left(x_{0}, A_{r}(Q)\right)} .
$$

Letting $D \ni y \rightarrow Q$, we get

$$
M_{D}(x, Q) \leq c_{4} \frac{G_{D}\left(x, A_{r}(Q)\right)}{G_{D}\left(x_{0}, A_{r}(Q)\right)}=c_{5} G_{D}\left(x, A_{r}(Q)\right), \quad x \in D \backslash B(Q, 2 r) .
$$

Recall that $\lim _{D \ni x \rightarrow z} G_{D}(x, y)=0$ for every regular $z \in \partial D$. Since $r<c / 4$ can be arbitrarily small, we see from (3.45) and (3.43) that $\lim _{D \ni x, x \rightarrow z} u(x)=0$ for every regular $z \in \partial D, z \neq Q$.

Assume $D$ is bounded. Fix $r<c / 4$. It follows from Lemma 2.12 that for all $x \in D \backslash B(Q, 2 r)$,

$$
G_{D}\left(x, A_{r}(Q)\right) \leq c_{6} \frac{\Phi\left(\left|x-A_{r}(Q)\right|\right)}{\left|x-A_{r}(Q)\right|^{d}} \leq c_{6} \sup _{a \geq r} \frac{\Phi(a)}{a^{d}} \leq c_{7}
$$

From (3.45) and (3.43) we conclude that $u$ is bounded in $x \in D \backslash B(Q, 2 r)$. Similarly, for every $x \in D \cap B(Q, c / 2)$ we have that $G_{D}\left(x, y_{0}\right) \leq c_{8} \sup _{a \geq c / 2} \Phi(c) c^{-d}=: c_{9}$ (recall $y_{0} \in D \backslash B(Q, c)$ ). Hence by (3.44) and (3.43) we see that $u$ is bounded on $D \cap B(Q, c / 2)$. Thus $u$ is bounded on $D$. Now it follows from Lemma 3.11 (a) that $u \equiv 0$ in $D$.

If $D$ is unbounded, we argue as follows. Since $G_{D}\left(x, A_{r}(Q)\right) \leq G\left(x, A_{r}(Q)\right)$, it follows from (3.44) and Lemma 2.20 that $\lim _{D \ni x \rightarrow \infty} M_{D}(x, Q)=0$. Hence by (3.43) $\lim _{D \ni x \rightarrow \infty} u(x)=0$. Thus, there exists $R \geq 2$ such that $u(x) \leq 1$ for all $x \in D \backslash B(Q, R)$. Fix $r<c / 4 \wedge 1$ and let $x \in D \cap(B(Q, R) \backslash B(Q, 2 r))$. By (3.45) and Theorem 2.19,

$$
M_{D}(x, Q) \leq c_{5} G\left(x, A_{r}(Q)\right) \leq c_{5} C_{2}(R) \frac{\Phi\left(\left|x-A_{r}(Q)\right|\right)}{\left|x-A_{r}(Q)\right|^{d}} \leq c_{10} \sup _{a \geq r} \frac{\Phi(a)}{a^{d}} \leq c_{11} .
$$


It follows that $u$ is bounded in $D \cap(B(Q, R) \backslash B(Q, 2 r))$. The proof that $u$ is bounded on $D \cap$ $B(Q, c / 2)$ is the same as in the case of a bounded $D$. Hence, $u$ is bounded, and again we conclude from Lemma 3.11 (b) that $u \equiv 0$ in $D$.

We see from (3.42) that $\nu=\mu_{\mid K_{\epsilon}}=0$. Since $\epsilon>0$ was arbitrary and $\partial_{M} D \backslash\{Q\}=\cup_{\epsilon>0} K_{\epsilon}$, we see that $\mu_{\mid \partial_{M} D \backslash\{Q\}}=0$. Hence $h=\mu(\{Q\}) M_{D}(\cdot, Q)$ showing that $M_{D}(\cdot, Q)$ is minimal.

In the next two results we assume that $D$ is a $\kappa$-fat set. Then one can define $\Xi: \partial D \rightarrow \partial_{M}^{f} D$ so that $\Xi(Q)$ is the unique element of $\partial_{M}^{Q} D$, cf. Proposition 3.6 .

Theorem 3.13 Suppose that either $D$ is bounded, or $D$ is unbounded and $X$ in transient. If $D$ is a $\kappa$-fat set, then the finite part of the minimal Martin boundary of $D$ and the finite part of the Martin boundary of $D$ both coincide with the Euclidean boundary $\partial D$ of D. More precisely, $\Xi$ is 1-1- and onto.

Proof. Since every finite Martin boundary point is associated with some $Q \in \partial D$, we see that $\Xi$ is onto. We show now that $\Xi$ is 1-1. If not, there are $Q, Q^{\prime} \in \partial D, Q \neq Q^{\prime}$, such that $\Xi(Q)=$ $\Xi\left(Q^{\prime}\right)=w$. Then $M_{D}(\cdot, Q)=M_{D}(\cdot, w)=M_{D}\left(\cdot, Q^{\prime}\right)$. Choose $r>0$ small enough and satisfying $r<\left|Q-Q^{\prime}\right| / 4$. By (3.45) and (3.46) we see that there exists a constant $c_{1}=c_{1}(Q)$ such that $M_{D}(x, Q) \leq c_{1}$ for all $x \in D \backslash B(Q, 2 r)$. Similarly, there exists $c_{2}=c_{2}\left(Q^{\prime}\right)$ such that $M_{D}\left(x, Q^{\prime}\right) \leq c_{2}$ for all $x \in D \backslash B\left(Q^{\prime}, 2 r\right)$. Since $B(Q, 2 r)$ and $B\left(Q^{\prime}, 2 r\right)$ are disjoint, we conclude that $M_{D}(\cdot, Q)=$ $M_{D}\left(\cdot, Q^{\prime}\right)$ is bounded on $D$ by $c_{1} \vee c_{2}$. Again by (3.45), $\lim _{D \ni x \rightarrow z} M_{D}(x, Q)=0$ for all regular $z \in$ $\partial D$. In case of unbounded $D$, we showed in the proof of Theorem 3.12 that $\lim _{x \rightarrow \infty} M_{D}(x, Q)=0$. Hence by Lemma 3.11 we conclude that $M_{D}(\cdot, Q) \equiv 0$. This is a contradiction with $M_{D}\left(x_{0}, Q\right)=1$.

The statement about the minimal Martin boundary follows from Theorem 3.12 .

As a consequence of the result above and the general result of [25]), we have the following Martin representation for nonnegative harmonic functions with respect to the killed process $X^{D}$.

Theorem 3.14 Suppose that $D$ is a bounded $\kappa$-fat set. Then $\Xi: \partial D \rightarrow \partial_{M} D$ is a homeomorphism. Furthermore, for any nonnegative function $u$ which is harmonic with respect to $X^{D}$, there exists a unique finite measure $\mu$ on $\partial D$ such that

$$
u(x)=\int_{\partial D} M_{D}(x, z) \mu(d z), \quad x \in D .
$$

Proof. Let $Q \in \partial D$ and $x \in D$. Choose $r<\frac{1}{2} \min \left\{R\right.$, $\operatorname{dist}(x, Q)$, $\left.\operatorname{dist}\left(x_{0}, Q\right)\right\}$ so that $x \in$ $D \backslash B(Q, 2 r)$. Let $Q^{\prime} \in \partial D \cap B(Q, r / 2)$. Since $D$ is $\kappa$-fat at $Q^{\prime}$, by Corollary 3.5 there exists $M_{D}\left(x, Q^{\prime}\right)=\lim _{D \ni y \rightarrow Q^{\prime}} M_{D}(x, y)$. Further, by letting $y \rightarrow Q^{\prime}$ in (3.34) we get that

$$
\left|M_{D}\left(x, Q^{\prime}\right)-M_{D}(x, Q)\right| \leq c M_{D}\left(x, A_{r}(Q)\right)\left(\frac{\left|Q^{\prime}-Q\right|}{r}\right)^{\beta} .
$$


This shows that if $\left(Q_{n}\right)_{n \geq 1}$ is a sequence of points in $\partial D$ converging to $Q \in \partial D$, then $M_{D}(\cdot, Q)=$ $\lim _{n \rightarrow \infty} M_{D}\left(\cdot, Q_{n}\right)$.

In order to show that $\Xi$ is continuous we proceed as follows. Let $Q_{n} \rightarrow Q$ in $\partial D$. Since $\partial_{M} D$ is compact, $\left(\Xi\left(Q_{n}\right)\right)_{n \geq 1}$ has a subsequence $\left(\Xi\left(Q_{n_{k}}\right)\right)_{k \geq 1}$ converging in the Martin topology to some $w \in \partial_{M} D$. By property $(\mathrm{M} 3), M_{D}\left(\cdot, \Xi\left(Q_{n_{k}}\right)\right) \rightarrow M_{D}(\cdot, w)$. On the other hand, by the first part of the proof, $M_{D}\left(\cdot, \Xi\left(Q_{n_{k}}\right)\right)=M_{D}\left(\cdot, Q_{n_{k}}\right) \rightarrow M_{D}(\cdot, Q)$, implying that $w=\Xi(Q)$. This shows in fact that $\left(\Xi\left(Q_{n}\right)\right)_{n \geq 1}$ is convergent with the limit $\Xi(Q)$. Using the fact that $\partial D$ is compact, the proof of the continuity of the inverse is similar.

The Martin representation for nonnegative harmonic functions is now a consequence of the general result form [25].

Acknowledgements. We thank the referee for helpful comments on the first version of this paper.

\section{References}

[1] C. Berg and G. Forst: Potential Theory on Locally Compact Abelian Groups. Springer, 1975.

[2] K. Bogdan: The boundary Harnack principle for the fractional Laplacian. Studia Math. 123(1)(1997), $43-80$.

[3] K. Bogdan: Representation of $\alpha$-harmonic functions in Lipschitz domains. Hiroshima Math. J. 29 (1999), 227-243.

[4] K. Bogdan, T. Grzywny and M. Ryznar: Density and tails of unimodal convolution semigroups. $J$. Funct. Anal. 266 (2014), 3543-3571.

[5] K. Bogdan, T. Grzywny and M. Ryznar: Barriers, exit time and survival probability for unimodal Lévy processes. Preprint. arXiv:1307.0270 [math.PR]

[6] K. Bogdan, T. Kulczycki, M. Kwaśnicki: Estimates and structure of $\alpha$-harmonic functions. Probab. Theory Rel. Fields. 140 (2008), 345-381.

[7] Z.-Q. Chen, P. Kim and T. Kumagai: On heat kernel estimates and parabolic Harnack inequality for jump processes on metric measure spaces. Acta Mathematica Sinica, English Series 25 (2009), 10671086.

[8] Z.-Q. Chen, P. Kim and R. Song: Dirichlet heat kernel estimates for fractional Laplacian with gradient perturbation. Ann. Probab. 40 (2012), 2483-2538.

[9] Z.-Q. Chen, P. Kim and R. Song: Dirichlet heat kernel estimates for rotationally symmetric Lévy processes. To appear in Proc. London Math. Soc.

[10] Z.-Q. Chen and T. Kumagai: Heat kernel estimates for jump processes of mixed types on metric measure spaces. Probab. Theory Relat. Fields, 140 (2008), 277-317.

[11] Z.-Q. Chen and R. Song: Martin boundary and integral representation for harmonic functions of symmetric stable processes. J. Funct. Anal. 159 (1998), 267-294.

[12] K.-L. Chung: Lectures from Markov Processes to Brownian Motion. Springer, New York (1982) 
[13] T. Grzywny: On Harnack inequality and Hölder regularity for isotropic unimodal Lévy processes. To appear in Potential Anal.

[14] R. A. Hunt and R. L. Wheeden: Positive harmonic functions on Lipschitz domains. Trans. Amer. Math. Soc. 147 (1970), 507-527.

[15] P. Kim and R. Song: Two-sided estimates on the density of Brownian motion with singular drift. Illinois J. Math., 50 (3), (2006), 635-688.

[16] P. Kim and R. Song: Boundary behavior of harmonic functions for truncated stable processes. $J$. Theoret. Probab. 21 (2008), 287-321.

[17] P. Kim, R. Song and Z. Vondraček: Boundary Harnack principle for subordinate Brownian motions. Stoch. Processee Appl. 119 (2009), 1601-1631.

[18] P. Kim, R. Song and Z. Vondraček: Potential theory of subordinate Brownian motions revisted. Analysis applications to finance, essays in honour of Jia-an Yan. Interdisciplinary Mathematical Sciences - Vol. 13, World Scientific, 2012, 277-317.

[19] P. Kim, R. Song and Z. Vondraček: Minimal thinness for subordinate Brownian motion in half space. Ann. Inst. Fourier 62 (3) (2012), 1045-1080.

[20] P. Kim, R. Song and Z. Vondraček: Two-sided Green function estimates for killed subordinate Brownian motions. Proc. London Math. Soc. 104 (2012), 927-958.

[21] P. Kim, R. Song and Z. Vondraček: Uniform boundary Harnack principle for rotationally symmetric Lévy processes in general open sets. Science China Math. 55 (2012), 2317-2333.

[22] P. Kim, R. Song and Z. Vondraček: Global uniform boundary Harnack principle with explicit decay rate and its application. Stoch. Proc. Appl. 124(1) (2014), 235-267.

[23] P. Kim, R. Song and Z. Vondraček: Boundary Harnack principle and Martin boundary at infinity for subordinate Brownian motions. To appear in Potential Analysis

[24] M. Kwaśnicki, J. Małecki, M. Ryznar: Suprema of Lévy processes. Ann. Probab. 41 (2013) 2047-2065.

[25] H. Kunita and T. Watanabe: Markov processes and Martin boundaries I. Illinois J. Math. 9(3) (1965) $485-526$.

[26] R.S. Martin: Minimal harmonic functions. Trans. Amer. Math. Soc. 49 (1941), 137-172.

[27] K. Michalik and K. Samotij: Martin representation for $\alpha$-harmonic functions. Probab. Math. Statist. 20 (2000), 75-91.

[28] R. Song, Z. Vondraček: Potential theory of subordinate Brownian motion. In: Potential Analysis of Stable Processes and its Extensions, P. Graczyk, A. Stos, editors, Lecture Notes in Mathematics 1980, (2009) 87-176.

[29] R. Song and J. Wu: Boundary Harnack principle for symmetric stable processes. J. Funct. Anal. 168 (1999), 403-427.

[30] P. Sztonyk: On harmonic measure for Lévy processes. Probab. Math. Statist. 20 (2000), 383-390.

[31] P. Sztonyk: Boundary potential theory for stable Lévy processes. Colloq. Math. 95 (2003), 191-206.

\section{Panki Kim}

Department of Mathematical Sciences and Research Institute of Mathematics, Seoul National University, Building 27, 1 Gwanak-ro, Gwanak-gu Seoul 151-747, Republic of Korea 
E-mail: pkim@snu.ac.kr

\section{Renming Song}

Department of Mathematics, University of Illinois, Urbana, IL 61801, USA

E-mail: rsong@math.uiuc.edu

\section{Zoran Vondraček}

Department of Mathematics, University of Zagreb, Zagreb, Croatia

Email: vondra@math.hr 